Pacific Journal of Mathematic 


\section{CONCERNING THE COHOMOLOGY RING OF A SPHERE BUNDLE}

\section{G MALM}

1. Introduction. This paper is concerned with the problem of determining the cohomology ring of an orientable fibre space whose fibere is a sphere, in terms of the cohomology ring of the base space and invariants of the fibre space.

When the fibering sphere is of even dimension $k-1$, an invariant $P$ in the $(2 k-2)$-dimensional cohomology group of the base space is defined, which is closely related to one of the Pontrjagin characteristic classes if the fibre space is a fibre bundle. If the $(2 k-2)$-dimensional cohomology group of the base space $B$ has no elements of order two, then two $(k-1)$-sphere spaces over $B$ with the same Stiefel-Whitney classes $W_{k}$ and $\mathrm{W}_{k-1}$ and the same invariant $P$ have isomorphic integral cohomology rings.

In the other case, when $k$ is even, if $H^{2 k-2}(B, Z)$ has no twotorsion, then two $(k-1)$-sphere spaces over $B$ with the same StiefelWhitney classes $W_{k}, W_{k-1}$, and $W_{k-2}$ have isomorphic integral cohomology rings.

If $H^{2 k-2}(B, Z)$ has elements of order two the situation seems to be more complicated and no results are obtained. Also, the problem of determining the cohomology ring with mod 2 coefficients is not touched upon here.

The method is based upon the algebraic mapping cylinder of the map $x \rightarrow x \mathscr{C}$, where $\mathscr{Z}$ is Thom's class, and thus parallels Thom's construction of the Gysin sequence using the mapping cylinder.

In conclusion, I wish to thank Professor W. S. Massey for his generous advice and encouragement in the preparation of this paper, which contains the essential parts of a dissertation submitted to Brown University.

2. Notation and terminology. We define a fibre space as an ordered quadruple $(E, p, B, F)$ such that $E, B$, and $F$ are topological spaces, $p: E \rightarrow B$ is a continuous map, and such that the following condition holds: For each $x \in B$, there is a neighborhood $U$ of $x$ and a homeomorphism $\phi$ mapping $U \times F$ onto $p^{-1}(U)$ such that $(p \phi)(y, z)=y$ for each $y \in U$ and $z \in F$. We call $E$ the total space, $F$ the fibre, and $B$ the base space.

By a fibre bundle is meant a fibre space with a structural group, as defined in Steenrod's book [8]. A fibre bundle whose fibre is an

Received January 19, 1959. The author received financial and other support from the National Science Foundation under Grant G1348 during the preparation of part of this paper. 
$n$-sphere and whose group is the group of all $(n+1) \times(n+1)$ real orthogonal matrices of determinant +1 (denoted by $S O(n+1)$ ) will be called an $n$-sphere bundle. An $n$-sphere space is a fibre space whose fibre is an $n$-sphere.

We assume that all $n$-sphere spaces with which we are concerned satisfy the following orientability condition: If $S_{x}^{n}$ denotes the fibre over the point $x \in B$, then the local system of groups defined by $H^{n}\left(S_{x}^{n}\right)$, for $x \in B$, is a simple system.

We also assume that the base space of any fibre space or fibre bundle we consider is compact, and we will use Cech-Alexander-Spanier cohomology with compact supports. Unless otherwise indicated, all cohomology groups are with integer coefficients.

In [11], R. Thom showed that the Gysin sequence of a $(k-1)$ sphere space $\left(E, p, B, S^{k-1}\right)$ may be obtained in the following manner: There is associated to the given $(k-1)$-sphere space another fibre space $\left(A, p_{0}, B, F\right)$ whose fibre $F$ is a $k$-cell, for which we may suppose $E \subset A$. ( $A$ is the mapping cylinder [10] of $p: E \rightarrow B)$. Thom showed that there is an element $\mathscr{U} \in H^{k}(A-E)=H^{k}(A, E)$ such that the homomorphism $\theta: H^{q-k}(A) \rightarrow H^{q}(A-E)$ defined by

$$
\theta(x)=x \mathscr{U}
$$

(the cup product) is an isomorphism onto. In addition $p_{0}^{*}: H^{q}(B) \rightarrow H^{q}(A)$ is an isomorphism onto. In fact, there is a cross section $s: B \rightarrow A$ where $s(x)$ is the center point of the fibre over $x$, and $s^{*}$ and $p_{0}^{*}$ are inverse to each other. We thus obtain the following commutative diagram of exact sequences, where all the vertical arrows are isomorphisms onto :

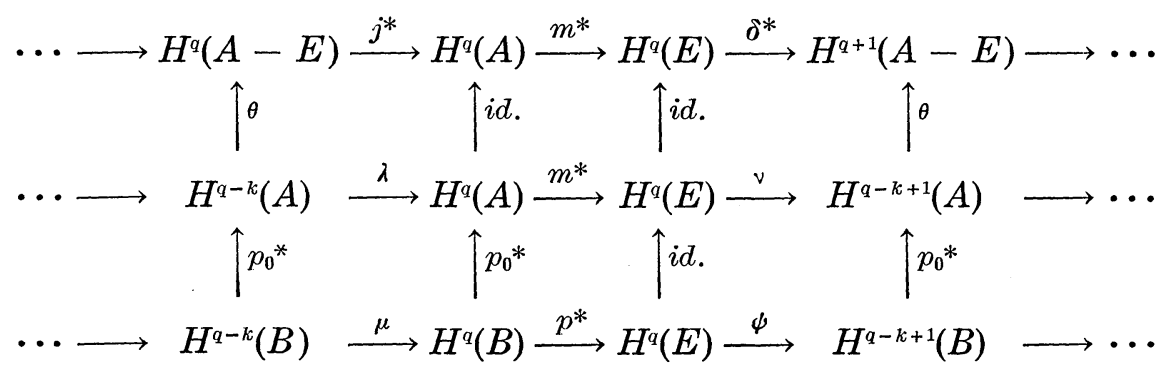

Figure 1

Here the homomorphisms $\lambda, \mu, \nu$, and $\psi$ are defined by $\lambda=j^{*} \theta$; $\mu=p_{0}^{*-1} \lambda p_{0}^{*} ; \nu=\theta^{-1} \delta^{*}$; and $\psi=p_{0}^{*-1} \nu$; the top horizontal sequence is the cohomology sequence of the pair $(A, E)$, and the bottom sequence is the Gysin sequence. Thus according to the results of Thom, the Gysin sequence of $\left(E, p, B, S^{k-1}\right)$ is isomorphic to the cohomology sequence of the pair $(A, E)$. 
In addition, if we let

and

$$
\begin{aligned}
w_{k} & =j^{*}(\mathscr{U}) \\
W_{k} & =p_{0}^{*-1}\left(w_{k}\right),
\end{aligned}
$$

then $W_{k}$ is the $k$ th Stiefel-Whitney class (the characteristic class)

and

$$
\begin{array}{ll}
\mu(x)=x W_{k} & \text { for } \quad x \in H^{*}(B), \\
\lambda(y)=y w_{k} & \text { for } \quad y \in H^{*}(A) .
\end{array}
$$

Define $w_{i}$ by

$$
\theta\left(w_{i}\right)=S_{q}^{i}(\mathscr{Q})
$$

where $S_{q}^{i}: H^{k}(A-E) \rightarrow H^{k+i}(A-E)$ denotes the Steenrod squaring operation (see [9], or [3], expose 14). ${ }^{*}$ Then also the Stiefel-Whitney classes $W_{i}$ are given by

$$
W_{i}=p_{0}^{*-1}\left(w_{i}\right) \text {. }
$$

Thus $W_{i} \in H^{i}(B, Z)$ for $i$ odd and $W_{i} \in H^{i}\left(B, Z_{2}\right)$ for $i$ even and less than $k . W_{k}$ is always an integral cohomology class. In addition, $2 W_{i}=0$ for $i$ odd. For more details, the reader is again referred to the paper of Thom [11].

We will regard $C^{*}(A, E)$ as a subgroup of $C^{*}(A)$. It is actually a two-sided ideal in $C^{*}(A)$, with respect to both the cup product and Steenrod's cup- $i$ products [9]. Since $C^{*}(E) \approx C^{*}(A) / C^{*}(A, E)$ (we are using Alexander-Čech cohomology), we identify these two cochain rings. Note that the map $j^{*}$ of Figure 1 is then induced by the inclusion $C^{*}(A, E) \subset C^{*}(A)$.

The notation introduced here will remain constant, for example, $A$ will always be the mapping cylinder of $p: E \rightarrow B$, $\mathscr{C}$ will always be the cohomology class introduced by Thom, etc.

Another important property of the Stiefel-Whitney classes is the following: The Bockstein homomorphism maps the even dimensional ones onto the odd dimensional ones (see [8], p. 195).

Finally, the map $\nu$ of Figure 1 satisfies the following equations: If $x \in H^{q}(A)$ and $y \in H^{p}(E)$, then

and

$$
\begin{aligned}
& \nu\left[m^{*}(x) \cdot y\right]=(-1)^{q} x \cdot(\nu y) \\
& \nu\left[y \cdot m^{*}(x)\right]=(-1)^{k g}(\nu y) \cdot x .
\end{aligned}
$$

This is Lemma 1 of [7].

3. The algebraic mapping cylinder of $\theta$. If $\left(E, p, B, S^{k-1}\right)$ is a sphere space, then using $p: H^{*}(B) \rightarrow H^{*}(E), H^{*}(E)$ is a module over $H^{*}(B)$ with the definition $x \cdot y=\left(p^{*} x\right) y$ for $x \in H^{*}(B)$ and $y \in H^{*}(E)$. The

* Here, for $i$ even, we let $\theta$ operate on $H^{*}\left(A, Z_{2}\right)$ in the obvious way. 
following is an unpublished result of $A$. Shapiro: If $\left(E, p, B, S^{k-1}\right)$ and $\left(E^{\prime}, p^{\prime}, B, S^{k-1}\right)$ are $(k-1)$-sphere spaces over the same base space $B$ with the same characteristic class $W_{k}$, then $H^{*}(E)$ and $H^{*}\left(E^{\prime}\right)$ are isomorphic as $H^{*}(B)$-modules. According to W. S. Massey, this may be proved in the following manner.

Let $\mathrm{A}$ be the mapping cylinder of $p: E \rightarrow B$ and let $U \in C^{*}(B)$ be such that $p_{0}^{\sharp} U \in \mathscr{U} \in H^{k}(A-E)$. Let $M$ be the algebraic mapping cylinder (see [5], page 159, Exercise D) of the map $x \rightarrow x U$ for $x \in C^{*}(B)$, that is

$$
\begin{aligned}
M^{p} & =C^{p}(B) \times C^{p-k+1}(B), \\
M & =\Sigma_{p} M^{p},
\end{aligned}
$$

and

$$
\delta(x, y)=(\delta x+y U,-\delta y) \text { for }(x, y) \in M .
$$

It is easily seen that $(M, \delta)$ is a differential group. In the diagram of exact sequences

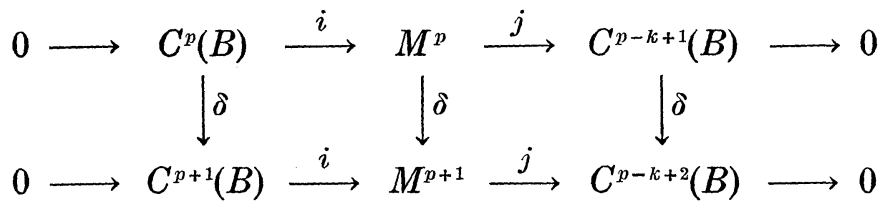

where $i(x)=(x, 0)$ and $j(x, y)=y$, the left square commutes and the right square anti-commutes. We obtain the exact sequence

$$
H^{p-k}(B) \stackrel{\mu}{\longrightarrow} H^{p}(B) \stackrel{i^{*}}{\longrightarrow} H^{p}(M) \stackrel{j^{*}}{\longrightarrow} H^{p-k+1}(B) \stackrel{\mu}{\longrightarrow} \cdots,
$$

where $\mu$ is the map induced by $x \rightarrow x U$, in other words, $\mu$ is the map $\mu$ of Figure 1 .

Now define $\eta: M^{p} \rightarrow C^{p}(A) / C^{p}(A, E)=C^{p}(E)$ by letting $\eta(x, y)$ be the equivalence class of $p_{0}^{\sharp}(x)$ in $C^{p}(A) / C^{p}(A, E)$. $\eta$ commutes with $\delta$ and thus induces $\eta^{*}: H^{p}(M) \rightarrow H^{p}(E)$. We then have the diagram

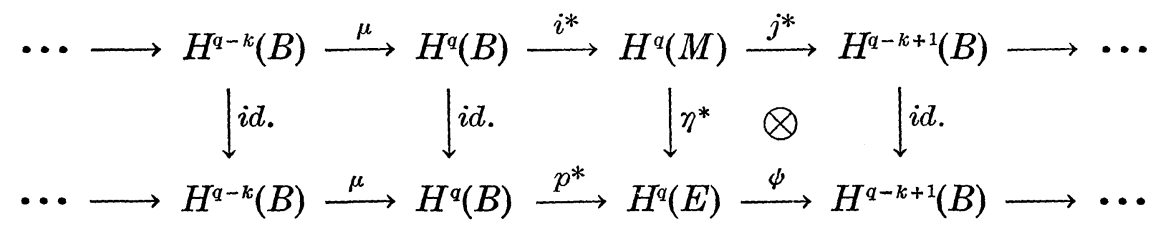

It is easily verified that all the squares commute except the square marked " $\otimes$ ", which anti-commutes. By the five-lemma, $\eta^{*}$ is an isomorphism onto.

We now make $M$ into a module over $C^{*}(B)$ by the definition

$$
x(v, w)=\left(x v,(-1)^{p} x w\right)
$$

for $x \in C^{p}(B)$ and $(v, w) \in M$. It is easily verified that $\delta\{x(v, w)\}=$ $(\delta x)(v, w)+(-1)^{p} x \delta(v, w)$ and thus $H^{*}(M)$ is an $H^{*}(B)$-module. For 
$x \in H^{*}(B)$ and $y \in H^{*}(M)$, we have $\eta^{*}(x y)=\left(p^{*} x\right)\left(\eta^{*} y\right)$, that is, $\eta^{*}$ preserves the module product. Consequently $H^{*}(M)$ and $H^{*}(E)$ are isomorphic as $H^{*}(B)$-modules.

Now suppose $U^{\prime}$ is any other representative for $W_{k}$. Then the maps $x \rightarrow x U$ and $x \rightarrow x U^{\prime}$ are chain homotopic, and it is easily seen that both algebraic mapping cylinders have isomorphic cohomology as $H^{*}(B)$-modules. Consequently, both $H^{*}(E)$ and $H^{*}\left(E^{\prime}\right)$ are isomorphic as $H^{*}(B)$-modules to $H^{*}(M)$, where $M$ is the algebraic mapping cylinder obtained from any representative for $W_{k}$.

We remark that it is misleading to say that " $H^{*}(E)$ depends only upon $H^{*}(B)$ and the characteristic class $W_{k}$." It is possible to give examples of polyhedra $B_{1}$ and $B_{2}$ such that their integral cohomology rings are isomorphic, and then construct 1-sphere bundles $\left(E_{1}, p_{1}, B_{1} S^{1}\right)$ and $\left(E_{2}, p_{2}, B_{2} S^{1}\right)$ such that the characteristic classes of the bundles correspond under the isomorphism, yet $H^{*}\left(E_{1}, Z\right)$ and $H^{*}\left(E_{2}, Z\right)$ are not isomorphic. The reason is that $H^{*}\left(B_{1}, Z_{2}\right)$ and $H^{*}\left(B_{2}, Z_{2}\right)$ are nonisomorphic, hence the mod two Gysin sequences are non-isomorphic, and $H^{*}\left(E_{1}, Z_{2}\right)$ and $H^{*}\left(E_{1}, Z_{2}\right)$ and $H^{*}\left(E_{2}, Z_{2}\right)$ do not have the some additive structure.

The theorems that follow concerning the cohomology ring of the total space $E$ will be obtained by introducing a multiplication in the algebraic mapping cylinder and proving that under certain circumstances the map $\eta^{*}$ (or rather, a similar map) is a ring homomorphism. For simlicity, we will work with the cochains of $A$ instead of $B$.

4. Adjusted triples and the multiplication in the mapping cylinder. We wish to define a bilinear function (product) from $M^{p} \times M^{q}$ into $M^{p+q}$ which obeys the familiar coboundary formula

$$
\delta(\alpha \cdot \beta)=(\delta \alpha) \cdot \beta+(-1)^{p} \alpha \cdot(\delta \beta)
$$

for $\alpha \in M^{p}$ and $\beta \in M^{\alpha}$. The problem may be simplified by observing that if $(x, y)$ and $(v, w)$ are elements of $M$, we require that

$$
\begin{aligned}
(x, y) \cdot(v, w) & =[(x, 0)+(0, y)][(v, 0)+(0, w)] \\
& =(x, 0)(v, w)+(0, y)(v, 0)+(0, y)(0, w) .
\end{aligned}
$$

Thus we divide the problem into three simpler ones; for each of these products we require that the coboundary formula holds. Furthermore, we know what the first product should be, for we want to preserve the module structure. Thus we want $(x, 0)(v, w)=\left(x v,(-1)^{p} x w\right)$ for $(x, o) \in M^{p}$. By a careful study of the last two products, we arrive at the following definitions.

DeFinition 4.1. An adjusted triple $(U, W, N)$ for the sphere space $\left(E, p, B, S^{k-1}\right)$ is a triple of coohains of $A$ for which: 
(i) $U \in \mathscr{U} \in H_{k}(A-E)$,

(ii) $W$ is a cochain representing $w_{k-1}$,

(iii) $\delta N=U \smile_{1} U-W U, N \in C^{2 k-2}(A, E)$,

(iv) $\delta w=\left\{\begin{array}{l}0 \text { if } k \text { is even } \\ -2 U \text { if } k \text { is odd. }\end{array}\right.$

DeFinition 4.2. Let $(U, W, N)$ be adjusted and let $M$ be the algebraic mapping cylinder of the map $x \rightarrow x U$. For $(x, y) \in M^{p}$ and $(v, w) \in M^{q}$, we define

$$
\begin{aligned}
& (x, y) \cdot(v, w)= \\
& \quad\left(x v+(-1)^{p+q} y\left(U \smile_{1} v\right)+(-1)^{p+k q+k+1} y\left[(w U) \smile_{2} U\right]+(-1)^{p+k(q+1)} y w N,\right. \\
& \left.(-1)^{p} x w+(-1)^{k q} y v+(-1)^{k+(k+1) q} y\left(w \smile_{1} U\right)+(-1)^{k+(k+1) q} y w W\right) .
\end{aligned}
$$

We will now prove two propositions which will justify the above definitions.

Lemma 4.3. For any sphere space $\left(E, p, B, S^{k-1}\right)$, there exists an adjusted triple $(U, W, N)$.

THEOREM 4.4. The product of Definition 4.2 is a bilinear function from $M^{p} \times M^{q}$ to $M^{p+q}$ for which $\delta[(x, y)(v, w)]=[\delta(x, y)](v, w)+$ $\left[(-1)^{p}(x, y)\right] \delta(v, w) . \quad$ Consequently a product $H^{p}(M) \times H^{q}(M) \rightarrow H^{p+q}(M)$ is induced. In addition, the additive isomorphism $\eta^{*}: H^{p}(M) \rightarrow H^{p}(E)$ induced by $\eta: M^{p} \rightarrow C^{p}(A) / C^{p}(A, E)$ where $\eta(x, y)$ is the equivalence class of $x$ in $C^{p}(A) / C^{p}(A, E)$, preserves products.

We prove Lemma 4.3 first. Suppose $k$ is even. Let $W$ be any cocycle representing $w_{k-1}$ and let $U$ be any cocycle representing $\mathscr{Q} \in H^{k}(A-E)$. By equation (2.2), $U \smile_{1} U$ and $W U$ represent the same element of $H^{2 k-1}(A-E)$. Thus there is a cochain $N$ in $C^{*}(A, E)$ for which $\delta N=U \smile_{1} U-W U$.

Now suppose that $k$ is odd. It is known that in this case, if $\Delta: H^{k-1}\left(A, Z_{2}\right) \rightarrow H^{k}(A, Z)$ is the Bockstein homomorphism, then $\Delta\left(w_{k-1}\right)=$ $w_{k}=-w_{k}$. Let $U \in \mathscr{U} \in H^{k}(A-E)$. If $W^{1}$ is any integral cochain representing $w_{k-1}$, then there is a cochain $R \in C^{*}(A)$ for which $\delta W^{1}=$ $-2 U+\delta R$, whence $\left(\delta W^{1}\right) U=2 U^{2}+(\delta R) U$. From (2.2) we see that $U \smile_{1} U=W^{1} U+\delta N^{1}+2 Q$ for some $N^{1}, Q \in C^{*}(A, E)$. Taking the coboundary, we get $-2 U^{2}=\left(\delta W^{1}\right) U+\delta(2 Q)$ and so $\delta(2 Q)=\delta(-R U)$, that is $2 Q+R U$ is a cocycle of $C^{*}(A, E)$. Since the map $\theta$ of (2.1) is an isomorphism, there is a cocycle $X$ of $C^{*}(A)$ and a cochain $\Xi \in C^{*}(A, E)$ for which $2 Q+R U=X U+\delta \Xi$. Consequently $U \smile_{1} U=$ $\left(W^{1}+X-R\right) U+\delta\left(N^{1}+\Xi\right)$. By taking cohomology classes, we see that $W^{1}+X-R$ represents $w_{k-1}$, and $\left(U, W^{1}+X-R, N^{1}+\Xi\right)$ is adjusted. This completes the proof of Lemma 4.3. 
To prove Theorem 4.4, we note first that the product is clearly bilinear, since the cup product and the cup- $i$ products are. To prove the coboundary formula, we compute

$$
\begin{aligned}
& \delta[(x, y)(v, w)]=\left(\delta(x v)+(-1)^{p+q} \delta\left[y\left(U \smile_{1} v\right)\right]+(-1)^{p+k q+k+1} \delta\left\{y\left[(w U) \smile_{2} U\right]\right\}\right. \\
& \quad+(-1)^{p+k(q+1)} \delta(y w N)+(-1)^{p} x w U+(-1)^{k q} y v U+(-1)^{k+(k+1) q} y\left(w \smile_{1} U\right) U \\
& \quad+(-1)^{k+(k+1) q} y w W U,-\left\{(-1)^{p} \delta(x w)+(-1)^{k q} \delta(y v)\right. \\
& \left.\left.\quad+(-1)^{k+(k+1) q} \delta\left[y\left(w \smile_{1} U\right)\right]+(-1)^{k+(k+1) q} \delta(y w W)\right\}\right)
\end{aligned}
$$

and

$$
\begin{aligned}
& {[\delta(x, y)](v, w)+\left[(-1)^{p}(x, y)\right] \delta(v, w)=(\delta x+y U,-\delta y)(v, w)} \\
& \quad+(-1)^{p}(x, y)(\delta v+w U,-\delta w) \\
& =\left((\delta x+y U) v+(-1)^{p+q+1}(-\delta y)\left(U \smile_{1} v\right)+(-1)^{p+k q+k}(-\delta y)\left[(w U) \smile_{2} U\right]\right. \\
& \quad+(-1)^{p+1+k(q+1)}(-\delta y) w N,(-1)^{p+1}(\delta x+y U) w+(-1)^{k q}(-\delta y) v \\
& \left.\quad+(-1)^{k+(k+1) q}(-\delta y)\left(w \smile_{1} U\right)+(-1)^{k+(k+1) q}(-\delta y) w W\right) \\
& \quad+(-1)^{p}\left(x(\delta v+w U)+(-1)^{p+q+1} y\left(U \smile_{1}[\delta v+w U]\right)\right. \\
& \quad+(-1)^{p+k(q+1)+k+1} y\left[\left(-(\delta w) U \smile_{2} U\right]+(-1)^{p+k(q)} y(-\delta w) N,\right. \\
& (-1)^{p} x(-\delta w)+(-1)^{k(q+1)} y(\delta v+w U)+(-1)^{k+(k+1)(q+1)} y\left((-\delta w) \smile_{1} U\right) \\
& \left.\quad+(-1)^{k+(k+1)(q+1)} y(-\delta w) W\right) .
\end{aligned}
$$

Thus the difference of the first components is

$$
\begin{aligned}
& (-1)^{p+q} \delta\left[y\left(U \smile_{1} v\right)\right]+(-1)^{p+k q+k+1} \delta\left\{y\left[(w U) \smile_{2} U\right]\right\}+(-1)^{p+k(q+1)} \delta(y w N) \\
& \quad+(-1)^{k q} y v U+(-1)^{k+(k+1) q} y\left(w \smile_{1} U\right) U+(-1)^{k+(k+1) q} y w W U-y U v \\
& \quad+(-1)^{p+q+1}(\delta y)\left(U \smile_{1} v\right)+(-1)^{p+k q+k}(\delta y)\left[(w U) \smile_{2} U\right]+(-1)^{p+1+k(q+1)}(\delta y) w N \\
& \quad+(-1)^{q} y\left(U \smile_{1}(\delta v+w U)\right)+(-1)^{k(q+1)+k+1} y\left[(\delta w \cdot U) \smile_{2} U\right] \\
& \quad+(-1)^{k q} y(\delta w) N .
\end{aligned}
$$

We now use the formula

$$
\begin{aligned}
\delta\left(u \smile_{i} v\right)= & (-1)^{p+q-i} u \smile_{i-1} v+(-1)^{p q+p+q} v \smile_{i-1} u+(\delta u) \smile_{i} v \\
& +(-1)^{p} u \smile_{i} \delta v
\end{aligned}
$$

for $u$ a $p$-cochain and $v$ a $q$-cochain (see [9]), and a formula due to G. Hirsch [6],

$$
(u v) \smile_{1} w=u\left(v \smile_{1} w\right)+(-1)^{q(r+1)}\left(u \smile_{1} w\right) v
$$

where $v$ and $w$ are $q$ - and $r$-cochains respectively. Thus

$$
\begin{aligned}
& (-1)^{p+q} \delta\left[y\left(U \smile_{1} v\right)\right]=(-1)^{p+q}(\delta y)\left(U \smile_{1} v\right) \\
& \quad+(-1)^{q+k+1} y\left\{(-1)^{k} U \smile_{1}(\delta v)+(-1)^{q+k+1} U v+(-1)^{k+q+k q} v U\right\} .
\end{aligned}
$$

Also, 


$$
\begin{aligned}
& (-1)^{p+k(q+1)} \delta(y w N)=(-1)^{p+k(q+1)}(\delta y)(w N)+(-1)^{k q+1} y\{(\delta w) N \\
& \left.\quad+(-1)^{q+k+1} w\left(U \smile_{1} U-W U\right)\right\} .
\end{aligned}
$$

Consequently the difference of the first components is seen to reduce to

$$
\begin{aligned}
& (-1)^{p+k q+k+1} \delta\left\{y\left[(w U) \smile_{2} U\right]\right\}+(-1)^{k+(k+1) q} y\left(w \smile_{1} U\right) U \\
& \quad+(-1)^{p+k q+k}(\delta y)\left[(w U) \smile_{2} U\right]+(-1)^{q} y\left(U \smile_{1}(w U)\right) \\
& \quad+(-1)^{k(q+1)+k+1} y\left[(\delta w \cdot U) \smile_{2} U\right]+(-1)^{k q+k+q} y w\left(U \smile_{1} U\right) .
\end{aligned}
$$

Since

$$
\begin{aligned}
& (-1)^{p+k q+k+1} \delta\left\{y\left[(w U) \smile_{2} U\right]\right\}=(-1)^{p+k q+k+1}(\delta y)\left[(w U) \smile_{2} U\right] \\
& \quad+(-1)^{k q} y\left\{(-1)^{q+k+1}(w U) \smile_{1} U+(-1)^{q+k+1+k(q+1)} U \smile_{1}(w U)\right. \\
& \quad+\left((\delta w \cdot U) \smile_{2} U\right\}
\end{aligned}
$$

this difference becomes

$$
\begin{aligned}
& (-1)^{k+(k+1) q} y\left(w \smile_{1} U\right) U+(-1)^{k q+k+q} y w\left(U \smile_{1} U\right) \\
& \quad+(-1)^{k q+q+k+1} y\left[(w U) \smile_{1} U\right],
\end{aligned}
$$

which is zero by the formula of G. Hirsch.

On the other hand, the difference of the second components of the two expressions is

$$
\begin{aligned}
& (-1)^{k+(k+1) q+1} \delta\left[y\left(w \smile_{1} U\right)\right]+(-1)^{k+(k+1) q+1} \delta(y w W)+(-1)^{p} y U w \\
& \quad+(-1)^{k+(k+1) q}(\delta y)\left(w \smile_{1} U\right)+(-1)^{k+(k+1) q}(\delta y) w W \\
& \quad+(-1)^{p+k(q+1)+1} y w U+(-1)^{p+k+(k+1)(q+1)} y\left[(\delta w) \smile_{1} U\right] \\
& \quad+(-1)^{p+k+(k+1)(q+1)} y(\delta w) W .
\end{aligned}
$$

But

$$
\begin{aligned}
\delta\left[y\left(w \smile_{1} U\right)\right]= & (\delta y)\left(w \smile_{1} U\right)+(-1)^{p+k+1} y\left[(\delta w) \smile_{1} U+(-1)^{q} w U\right. \\
& \left.+(-1)^{q+1+(q+k+1) k} U w\right],
\end{aligned}
$$

and

$$
\delta(y w W)=(\delta y)(w W)+(-1)^{p+k+1} y\left[(\delta w) \cdot W+(-1)^{q+k+1} w(\delta W)\right] .
$$

Thus this difference reduces to

$$
\begin{gathered}
(-1)^{p+k(q+1)+1} y w U+(-1)^{p+k q} y w U+(-1)^{p+k(q+1)+1} y w(\delta W) \\
=(-1)^{p+k q}\left[(-1)^{k+1} y w U+y w U+(-1)^{k+1} y w \delta W\right]=0,
\end{gathered}
$$

since $\delta W=0$ for $k$ even and $\delta W=-2 U$ for $k$ odd.

Thus the cochain formula holds and a product is induced on the cohomology level. Since $C^{*}(A, E)$ is an ideal in $C^{*}(A)$, and since $U$ and $N$ are in $C^{*}(A, E)$, we see immediately from the definitions of $\eta$ and the product that $\eta^{*}$ preserves products. This completes the proof of Theorem 4.4. 
We remark that the product of Definition 4.2 is not associative, though, of course, the induced product on the cohomology level is.

5. The invariant $\boldsymbol{P}$. We now define, when $k$ is odd, an invariant $P^{\prime}$ of the $(k-1)$-sphere space $\left(E, p, B, S^{k-1}\right)$, which is an element of $H^{2 k-2}(A, Z)$, and its image $P=p_{0}^{*-1}\left(P^{\prime}\right) \in H^{2 k-2}(B, Z) . \quad P^{\prime}$ and $P$ will be called the $P^{\prime}$-invariant and the $P$-invariant, respectively, of the sphere space.

Let $(U, W, N)$ be an adjusted triple. A straightforward computation, using equation (4.5), shows that $W^{2}+W \smile_{1}(\delta W)-4 N-U \smile_{2} U$ is a $(2 k-2)$-cocycle. We define $P^{\prime}$ to be its cohomology class in $H^{2 k-2}(A, Z)$, and $P=p_{0}^{*-1}\left(P^{\prime}\right)$.

TheOREM 5.1. Let $\left(E, p, B, S^{k-1}\right)$ be a sphere space for which $k$ is odd, and let $(U, W, N)$ and $\left(U^{\prime}, W^{\prime}, N^{\prime}\right)$ be adjusted triples for this sphere space. Then $W^{2}+W \smile_{1}(\delta W)-4 N-U \smile_{2} U$ and $W^{\prime 2}+W^{\prime} \smile_{1}\left(\delta W^{\prime}\right)-$ $4 N^{\prime}-U^{\prime} \smile_{2} U^{\prime}$ represent the same element of $H^{2 k-2}(A)$, and consequently $P^{\prime}$ is independent of the choice of nice triple made in its definition.

This theorem, which states that $P$ is an invariant of the sphere space, is proved with the help of the following lemma.

Lemma 5.2. Let $k$ be odd, and let $(U, W, N)$ be an adjusted triple. Then $\left(U^{\prime}, W^{\prime}, N^{\prime}\right)$ is an adjusted triple if, and only if, there exist $\beta \in C^{k-1}(A, E), \gamma \in C^{k-2}(A), \phi \in C^{k-2}(A)$ a cocycle $\bmod 2$, and $\rho \in$ $C^{2 k-2}(A, E)$ a cocycle, for which

$$
\begin{aligned}
U^{\prime} & =U+\delta \beta, \\
W^{\prime} & =W-2 \beta+\delta(\varphi+\gamma),
\end{aligned}
$$

and

$$
N^{\prime}=N+\beta \smile_{1} U^{\prime}-U \smile_{1} \beta-(\varphi+\gamma) U^{\prime}+\beta^{2}-W \beta+\rho .
$$

We first prove that if $(U, W, N)$ and $\left(U^{\prime}, W^{\prime}, N^{\prime}\right)$ are adjusted, then there exist $\beta, \gamma, \varphi$, and $\rho$ with the stated properties. Since $U$ and $U^{\prime}$ both represent $\mathscr{U}$, there exists $\beta \in C^{k-1}(A, E)$ for which $U^{\prime}=U+\delta \beta$. Now $\delta W^{\prime}=-2 U^{\prime}=-2(U+\delta \beta)=\delta W-\delta(2 \beta)$, or $W^{\prime}-W+2 \beta$ is a cocycle. Let $\alpha^{\prime}=W^{\prime}-W+2 \beta$. Taking cohomology $\bmod 2$ in $A$, (denoted by brackets) we see that $0=\left[W^{\prime}-W\right]=\left[2 \beta-\alpha^{\prime}\right]=\left[\alpha^{\prime}\right]$. Thus there exist $\gamma \in C^{k-2}(A)$ and $\alpha \in C^{k-1}(A)$ for which $\alpha^{\prime}=\delta(\gamma)+2 \alpha$, and $\delta \alpha=0$. Then $W^{\prime}=W+2(\alpha-\beta)+\delta \gamma$. Now

$$
\begin{aligned}
\delta\left(N^{\prime}-N\right)= & U^{\prime} \smile_{1} U^{\prime}-U \smile_{1} U+W U-W^{\prime} U^{\prime} \\
= & (\delta \beta) \smile_{1} U+U \smile_{1}(\delta \beta)+(\delta \beta) \smile_{1}(\delta \beta)-2(\alpha-\beta) U \\
& -\delta(\gamma U)-W(\delta \beta)-2(\alpha-\beta) \delta \beta-\delta(\gamma \delta \beta) .
\end{aligned}
$$


Using equation (4.5), we have

$$
\delta\left(\beta \smile_{1} U-U \smile_{1} \beta\right)=(\delta \beta) \smile_{1} U+U \smile_{1}(\delta \beta)+2 \beta U-2 U \beta .
$$

Consequently

$$
\begin{aligned}
\delta\left(N^{\prime}-N\right)= & \delta\left(\beta \smile_{1} U-U \smile_{1} \beta\right)-\delta(W \beta)+(\delta \beta) \smile_{1}(\delta \beta)-2 \alpha U \\
& -\delta(\gamma U)-2 \alpha(\delta \beta)-\delta(\gamma \delta \beta)+2 \beta \delta \beta .
\end{aligned}
$$

But since $(\delta \beta) \smile_{1}(\delta \beta)=\delta\left(\beta \smile_{1}(\delta \beta)\right)-\beta \delta \beta+(\delta \beta) \beta$, we have

$$
\begin{aligned}
\delta\left(N^{\prime}-N\right)= & \delta\left\{\beta \smile_{1} U-U \smile_{1} \beta-W \beta-\gamma U+\beta^{2}-\gamma \delta \beta+\beta \smile_{1}(\delta \beta)\right\} \\
& -2 \alpha U^{\prime} \\
= & \delta\left\{\beta \smile_{1} U^{\prime}-U \smile_{1} \beta-\gamma U^{\prime}-W \beta+\beta^{2}\right\}-2 \alpha U^{\prime},
\end{aligned}
$$

which states that $2 \alpha U^{\prime}$ is a coboundary of $C^{*}(A, E)$, since $N^{\prime}, \beta, U^{\prime}, U$, and $N$ are in $C^{*}(A, E)$. Since $\theta$ of (2.1) is an isomorphism, this means that there is $\varphi \in C^{k-2}(A)$ such that $2 \alpha=\delta \varphi$, and $-2 \alpha U^{\prime}=-\delta\left(\varphi U^{\prime}\right)$. We then have

$$
\delta\left(N^{\prime}-N\right)=\delta\left(\beta \smile_{1} U^{\prime}-U \smile_{1} \beta-\gamma U^{\prime}-W \beta+\beta^{2}\right) .
$$

This gives the stated result immediately.

Now suppose $(U, W, N)$ is adjusted and $\beta, \gamma, \varphi$, and $\rho$ have the stated properties. Then clearly $U^{\prime}$ represents $\mathscr{C}$ and $W^{\prime}$ represents $w_{k-1}$. Also $\delta W^{\prime}=\delta W-2 \delta \beta=-2 U-2 \delta \beta=-2 U^{\prime}$. Finally,

$$
\begin{aligned}
\delta N^{\prime}= & U \smile_{1} U-W U+\delta\left(\beta \smile_{1} U^{\prime}\right)-\delta\left(U \smile_{1} \beta\right)-\delta(\varphi+\gamma) U^{\prime}+\beta \delta \beta \\
& +(\delta \beta) \beta-W \delta \beta+2 U \beta \\
= & U \smile_{1} U-W U^{\prime}-\delta(\varphi+\gamma) U^{\prime}+(\delta \beta) \smile_{1} U^{\prime}+\beta U^{\prime}-U^{\prime} \beta \\
& +U \smile_{1} \delta \beta-U \beta+\beta U+\beta \delta \beta+(\delta \beta) \beta+2 U \beta \\
= & U^{\prime} \smile_{1} U^{\prime}-W U^{\prime}-\delta(\varphi+\gamma) U^{\prime}+2 \beta U^{\prime} \\
= & U^{\prime} \smile_{1} U^{\prime}-W^{\prime} U^{\prime} .
\end{aligned}
$$

Consequently $\left(U^{\prime}, W^{\prime}, N^{\prime}\right)$ is adjusted. This completes the proof of Lemma 5.2.

We now prove Theorem 5.1. Let $(U, W, N)$ and $\left(U^{\prime}, W^{\prime}, N^{\prime}\right)$ be adjusted triples related by $\beta, \gamma, \varphi$, and $\rho$ as in Lemma 5.2. Let

$$
a=W^{2}+W \smile_{1}(\delta W)-4 N-U \smile_{2} U
$$

and

$$
a^{\prime}=W^{\prime 2}+W^{\prime} \smile_{1}\left(\delta W^{\prime}\right)-4 N^{\prime}-U^{\prime} \smile_{2} U^{\prime} .
$$

Then

$$
\begin{aligned}
a^{\prime}-a= & W^{\prime 2}-W^{2}+W^{\prime} \smile_{1}\left(\delta W^{\prime}\right)-W \smile_{1}(\delta W)+4\left(N-N^{\prime}\right) \\
& +U \smile_{2} U-U^{\prime} \smile_{2} U^{\prime} .
\end{aligned}
$$


Since $U \smile_{2} U$ and $U^{\prime} \smile_{2} U^{\prime}$ both represent $w_{k-2} \mathscr{U}$, we may let $\delta z=$ $U \smile_{2} U-U^{\prime} \smile_{2} U^{\prime}$, for some $z \in C^{*}(A, E)$. Then it is easily verified, using Lemma 5.2 and equation (4.5), that

$$
\begin{aligned}
a^{\prime}-a=-4 \rho+ & \delta\left\{z-(\varphi+\gamma) \smile_{1}\left(2 U^{\prime}\right)+W^{\prime}(\varphi+\gamma)\right. \\
& \left.-(\varphi+\gamma) \delta(\varphi+\gamma)+(\varphi+\gamma) W^{\prime}-W \smile_{1}(2 \beta)\right\} .
\end{aligned}
$$

Thus, taking cohomology in $H^{*}(A)$, we have $\left[a^{\prime}-a\right]=[-4 \rho]$. But $\rho$ is a cocycle of $A-E$ and thus for some $X \in H^{*}(A)$ we have $[\rho]=X \mathscr{U}$ where the cohomology class of $\rho$ is here taken in $H^{*}(A-E)$. Consequently, now taking cohomology classes in $H^{*}(A)$, we have $[-4 \rho]=$ $-4\left(X w_{k}\right)$ which is zero since $2 w_{k}=0$. This completes the proof of Theorem 5.1.

We now turn to some properties of $P$. We shall prove the following theorem:

Theorem 5.3. Let $k$ be odd, and let $\left(E, p, B, S^{k-1}, S O(k)\right)$ be a $(k-1)$ sphere bundle, with $B$ a finite polyhedron. If $H^{2 k-2}(B, Z)$ has no elements of order two, then $P=P_{2 k-2}$, the Pontrjagin class in dimension $2 k-2$.

The hypothesis that the fibre space admit $S O(k)$ as structural group is needed in order that the Pontrjagin class be defined.

The proof of this theorem requires several lemmas and the use of the universal Gysin sequence.

We recall [8] that given any topological group $G$, there exists a universal principal $G$-bundle $\left(E_{G}, p, B_{G}, G, G\right)$ which has the following property:

Given a polyhedron $B$, any principal $G$-bundle over $B$ is isomorphic to the bundle induced by some $\operatorname{map} f: B \rightarrow B_{G} . B_{G}$ is called the classifying space for $G$.

Suppose now that $G_{0}$ is a closed subgroup of $G$. The following lemma is proved by H. Cartan in [3], exposé 7 .

Lemma 5.4. If $(E, p, B, G, G)$ is a principal G-bundle, and $\pi: E / G_{0} \rightarrow$ $E / G=B$ is the natural projection, then $\left(E / G_{0}, \pi, B, G / G_{0}, G\right)$ is a fibre bundle which is associated with $(E, p, B, G, G)$, where $G$ operates on $G / G_{0}$ in the natural way.

It is known that if $\left(E_{G}, p, B_{G}, G, G\right)$ is a universal principal $G$-bundle, and $G_{0}$ is a closed subgroup of $G$, then in the associated fibre bundle $\left(E_{G} / G_{0}, \pi, B, G / G_{0}, G\right)$ given by Lemma 5.4 , the total space $E_{G} / G_{0}$ is of the same homotopy type as the classifying space $B_{G_{0}}$. For a proof, see [7], Lemma 6. Taking $G=S O(k), G_{0}=S O(k-1)$, we have $G / G_{0}=S^{k-1}$.

We will call $\left(B_{S O(k-1)}, \pi, B_{S o(k)}, S^{k-1}, S O(k)\right)$ the universal $(k-1)$ sphere bundle. It has the following pleasant property: Any bundle 
$\left(E, p, B, S^{k-1}, S O(k)\right)$ is isomorphic to the induced bundle $f^{-1}\left(B_{S O(k-1)}, \pi\right.$, $\left.B_{S O(k)}, S^{k-1}, S O(k)\right)$ for some map $f: B \rightarrow B_{S O(k)}$. This follows from the fact that the operation of taking induced bundles and of taking associated bundles commute. This is easily proved if one uses the definition of "induced bundle" and "associated bundle" in terms of the coordinate transformations ([8]).

Lemma 5.5. Let $\left(E^{\prime}, p^{\prime}, B^{\prime}, S^{k-1}, S O(k)\right)$ be a $(k-1)$-sphere bundle, with $P$-invariant $\beta^{\prime}$, and let $\left(E, p, B, S^{k-1}, S O(k)\right)$ be the bundle induced by $f: B \rightarrow B^{\prime}$, with $P$-invariant $\beta$. Then $f^{*}\left(\beta^{\prime}\right)=\beta$.

Proof. Let $F: E \rightarrow E^{\prime}$ be the map of the total spaces corresponding to $f$, so that the following diagram is commutative:

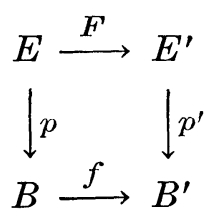

This diagram may be imbedded in a commutative diagram

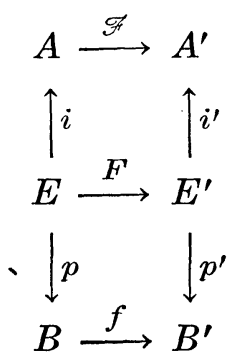

where $A$ and $A^{\prime}$ are the mapping cylinders of $p$ and $p^{\prime}$ respectively, and $i$ and $i^{\prime}$ are inclusion maps. $A$ is a quotient space of $(E \times I) \cup B$, where $I$ is the closed unit interval, and similarly for $A^{\prime}$. Letting square brackets denote equivalence classes in the quotient spaces, $\mathscr{F}$ is defined by

$$
\mathscr{F}[(x, t)]=[(F x, t)] \quad \text { for } x \in E, t \in I,
$$

and

$$
\mathscr{F}[b]=[f b] \quad \text { for } b \in B \text {. }
$$

Also $i(e)=[(e, 0)]$ for $e \in E$. It is easily verified that $\mathscr{F}$ is a continuous function and the diagram commutes. Let $\mathscr{F}^{*}: C^{*}\left(A^{\prime}\right) \rightarrow C^{*}(A)$ be the cochain homomorphism induced by $\mathscr{F}$.

Passing to the cochain level we have the commutative diagram 


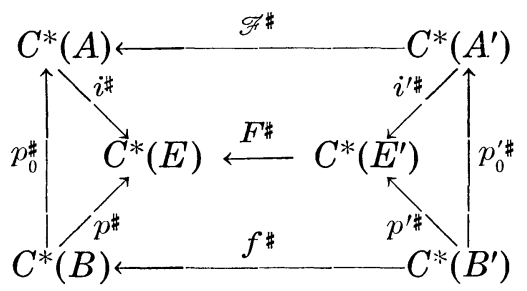

Figure 2

where $p_{0}: A \rightarrow B$ and $p_{0}^{\prime}: A^{\prime} \rightarrow B^{\prime}$ are the projections.

Now let $\left(U^{\prime}, W^{\prime}, N^{\prime}\right)$ be an adjusted triple for $\left(E^{\prime}, p^{\prime}, B^{\prime}, S^{k-1}\right)$. Let $U=\mathscr{F}^{\sharp}\left(U^{\prime}\right), W=\mathscr{F}^{\sharp}\left(W^{\prime}\right)$, and $N=\mathscr{F}^{\sharp}\left(N^{\prime}\right)$. Clearly $\mathscr{F}^{\sharp}\left(C^{*}\left(A^{\prime}, E^{\prime}\right)\right) \subset$ $C^{*}(A, E)$. Since the Stiefel-Whitney classes and Thom's class $\mathscr{U}$ are preserved by $f$ (or $\mathscr{F}$ ), we see that $(U, W, N)$ is an adjusted triple for $(E, p, B)$. A representative cocycle for the $P^{\prime}$-invariant of $\left(E^{\prime}, p^{\prime}, B^{\prime}\right)$ is $W^{\prime 2}+W^{\prime} \smile_{1} \delta W^{\prime}-4 N^{\prime}-U^{\prime} \smile_{2} U^{\prime}$, under $\mathscr{F}^{\#}$ this goes into $W^{2}+$ $W \smile_{1} \delta W-4 N-U \smile_{2} U$, a representative cocycle for the $P^{\prime}$-invariant of $(E, p, B)$. Consequently $f^{*}\left(\beta^{\prime}\right)=\beta$, in view of the commutativity of Figure 2.

The following two lemmas together imply Theorem 5.3.

LEMmA 5.6. Let $k$ be odd, and $\left(E, p, B, S^{k-1}, S O(k)\right) a(k-1)$-sphere bundle, with $B$ a finite polyhedron. Using the rationals or the integers $\bmod n, n$ odd, as coefficients for cohomology, $P=P_{2 k-2}$, the Pontrjagin class in dimension $2 k-2$ with rational or $\bmod n$ coefficients.

LEMMA 5.7. Let $G$ be a finitely generated abelian group with no elements of order two. Let $a \in G$ be such that for each odd integer $n$ there is an $\alpha \in G$ for which $a=n \alpha$. Then $a=0$.

We omit the proof of Lemma 5.7, which is quite simple.

Proof of Lemma 5.6. In view of Lemma 5.5, it suffices to prove Lemma 5.6 for the universal $(k-1)$-sphere bundle $\left(B_{S O(k-1)}, \pi, B_{S O(k)}\right.$, $\left.S^{k-1}, S O(k)\right)$.

Since the base space $B$ of our bundle is a finite polyhedron, we need only use an $n$-universal bundle for sufficiently large $n$. For this bundle, the base space may be chosen to be compact (see [8], Section 19), and we may use Alexander-Spanier cohomology with compact supports.

Let $W_{k}$ be the characteristic class of this bundle, thus $W_{k} \in H^{k}\left(B_{S O(k)}\right)$, and let $W_{k-1}(k-1) \in H^{k-1}\left(B_{S o(k-1)}\right)$ be the universal Euler-Poincaré class (for the cohomology of the classifying spaces see the article by A. Borel [1]; for a review of the results we need, see the article by W. S. Massey [7]). Since $k$ is odd, $2 W_{k}=0$ and $W_{k}=0$.

Choose $(U, W, N)$ adjusted for this sphere bundle and let $M$ be the 
algebraic mapping cylinder associated with this adjusted triple, with a multiplication defined as in $\S 4$. We then have the following commutative diagram of exact sequences, where all the vertical arrows are isomorphisms onto (the square marked " $\otimes$ " anticommutes). The notation is that used previously.

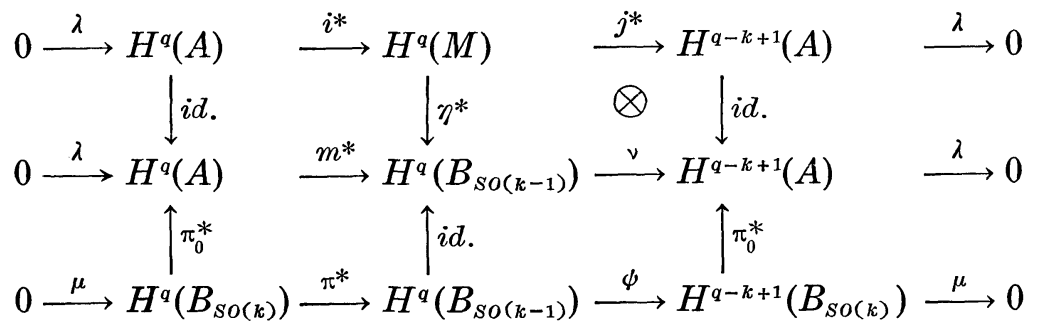

Fig. 3 .

In what follows, an integer $n$ is to be taken as $n \omega$ or $\bar{n} \omega^{\prime}$ if the coefficients are the rationals or the integers mod an odd integer respectively. Here, $\omega$ is the unit of $C^{*}(A$, rationals $), \omega^{\prime}$ is the unit of $C^{*}\left(A, Z_{2 m+1}\right)$, and $\bar{n}$ is $n$ reduced $\bmod 2 m+1$.

We note that $(W, 2)$ is a cocycle of $M^{k-1}$ and compute that $(W, 2)^{2}=$ $\left(W^{2}-(\delta W) \smile_{1} W+4 U \smile_{2} U-4 N, 0\right)$. Since $(\delta W) \smile_{1} W=\delta\left(W \smile_{1} W\right)-$ $W \smile_{1}(\delta W)$, we have

$$
(W, 2)^{2}=\left(Z+5 U \smile_{2} U-\delta\left(W \smile_{1} W\right), 0\right)
$$

where $Z=W^{2}+W \smile_{1}(\delta W)-4 N-U \smile_{2} U$ is a representative cocycle for the invariant $P^{\prime} \in H^{2 k-2}(A)$. Since $U$ is a coboundary, $5 U \smile_{2} U$ is and

$$
i^{*}\left(P^{\prime}\right)=\left[(W, 2)^{2}\right] \in H^{2 k-2}(M),
$$

where the square brackets denote cohomology classes. Thus $m^{*}\left(P^{\prime}\right)=$ $\left(\eta^{*} i^{*}\right)\left(P^{\prime}\right)=\eta^{*}[(W, 2)] \eta^{*}[(W, 2)]=[W]^{2} \in H^{k-1}\left(B_{S o(k-1)}\right)$, and $\left(\pi^{*} \circ \pi_{0}^{*-1}\right)\left(P^{\prime}\right)=$ $[W]^{2}$, or $\pi^{*}(P)=[W]^{2}$. We now need the following lemmas.

LEMMA 5.8. With integral coefficients, $\psi\left(W_{k-1}(k-1)\right)$ is twice a generator of $H^{0}\left(B_{S o(k)}\right)$.

LEMma 5.9. With integral coefficients, $\pi^{*}\left(P_{2 k-2}\right)=\left(W_{k-1}(k-1)\right)^{2}$.

For proofs, see [7], Lemmas 7 and 8.

Thus, using the rationals or the integers mod an odd integer for coefficients, we have $\pi^{*}\left(P_{2 k-2}\right)=\left(W_{k-1}(k-1)\right)^{2}$. Now $\pi^{*}$ is an isomorphism and we complete the proof that $P=P_{2 k-2}$ by showing that $\left(W_{k-1}(k-1)\right)^{2}=$ $[W]^{2}$.

By Lemma 5.8, we may choose $\varepsilon= \pm 1$ so that $\nu\left(\varepsilon W_{k-1}(k-1)\right)=$ $-2 \in H^{0}(A)$. But $\nu([W])=-j^{*}[(W, 2)]=-2$. By exactness there is a $y \in H^{*}(A)$ such that $\varepsilon W_{k-1}(k-1)=[W]+m^{*}(y)$. Multiplying by $[W]$ 
and $\varepsilon W_{k-1}(k-1)$ respectively, we get

$$
\varepsilon W_{k-1}(k-1)[W]=[W]^{2}+m^{*}(y)[W]
$$

and

$$
\left(W_{k-1}(k-1)\right)^{2}=\varepsilon W_{k-1}(k-1)[W]+m^{*}(y) \varepsilon W_{k-1}(k-1) .
$$

Together these give us

$$
[W]^{2}-\left(W_{k-1}(k-1)\right)^{2}=-m^{*}(y)\left([W]+\varepsilon W_{k-1}(k-1)\right) .
$$

We now apply $\nu$ to this equation, remembering that $[W]^{2}$ and $\left.W_{k-1}(k-1)\right)^{2}$ are in image $m^{*}=$ kernel $\nu$. Then

$$
0=-y \nu\left([W]+\varepsilon W_{k-1}(k-1)\right)=4 y .
$$

Thus $y=0$ and $\left(W_{k-1}(k-1)\right)^{2}=[W]^{2}$.

It is possible to prove the following theorem, which immediately implies Lemma 5.6.

If $k$ is odd, and $\left(E, p, B, S^{k-1}, S O(k)\right)$ is a $(k-1)$-sphere bundle with $W_{k}=0$ and $B$ a polyhedron, then $P=P_{2 k-2}$, the Pontrjagin class in dimension $2 k-2$.

This is a direct consequence of Theorem IV of [7]. It is only necessary to prove that $P$ is Massey's invariant $4 \alpha+\beta^{2}$, which can be done by a computation in the mapping cylinder.

According to W. T. Wu [12], for a $(k-1)$-sphere bundle, if $\mathscr{T}^{2}$ denotes the Pontrjagin squaring operation, then

$$
P_{2 k-2}=\mathscr{P}^{2}\left(W_{k-1}\right)-W_{k-2} W_{k},
$$

reduced $\bmod 4$. If $(U, W, N)$ is adjusted for the sphere bundle, $W^{2}+W \smile_{1}(\delta W)$ represents $\mathscr{P}^{2}\left(w_{k-1}\right)$ and $U \smile_{2} U$ represents $w_{k-2} w_{k}$. Consequently,

$$
P=P_{2 k-2} \text { reduced } \bmod 4 \text {. }
$$

Let $G_{k-1}$ denote the group of all homeomorphisms of $S^{k-1}$, and $B_{G(k-1)}$ the classifying space for $G_{k-1}$. It would be of interest to know whether the invariant $P$ comes from a cohomology class in $H^{2 k-2}\left(B_{G_{k-1}}\right)$.

6. The main theorem for $k$ odd. In this section we assume that $k$ is odd and $\left(E, p, B, S^{k-1}\right)$ is a $(k-1)$-sphere space. We consider the effect of dropping the conditions that $N$ and $U$ be in $C^{*}(A, E)$, where $(U, W, N)$ is an adjusted triple for $\left(E, p, B, S^{k-1}\right)$. A check of the proof of Theorem 4.4 shows that the product of Definition 4.2 still induces a product in the mapping cylinder. However, in general $\eta^{*}$ no longer preserves products. To retrieve (in part) this property of $\eta^{*}$ we add a 
requirement that $U, W$, and $N$ be connected with the invariant $P^{\prime}$. Before stating the main theorem we require several lemmas.

LeMma 6.1. Let $U \in w_{k} \in H^{k}(A), Z \in P^{\prime} \in H^{2 k-2}(A)$, and let $W$ be any integral cochain representing $w_{k-1}$ for which $\delta W=-2 U$. Then there exist $N \in C^{2 k-2}(A)$ and $\mathrm{Q} \in C^{2 k-3}(A)$ for which

$$
4 N+\delta Q=W^{2}+W \smile_{1}(\delta W)-Z-U \smile_{2} U .
$$

Proof. Let $\left(U^{\prime}, W^{\prime}, N^{\prime}\right)$ be adjusted, and let $Z^{\prime}=W^{\prime 2}+W^{\prime} \smile_{2}\left(\delta W^{\prime}\right)$ $-4 N^{\prime}-U^{\prime} \smile_{2} U$, a representative cocycle for $P^{\prime}$. Then there are cochains $\alpha, \beta$, and $\gamma$ for which $U=U^{\prime}+\delta \beta, W=W^{\prime}+\delta \gamma-2 \beta$, and $Z=Z^{\prime}+\delta \alpha$. Let

$$
N=N^{\prime}-W^{\prime} \beta+\beta \smile_{1} U^{\prime}+\beta^{2}-U^{\prime} \smile_{1} \beta-(\delta \gamma) \beta+\beta \smile_{1}(\delta \beta)-\gamma U^{\prime}
$$

and

$$
\begin{aligned}
Q=-\gamma \smile_{1}\left(2 U^{\prime}\right) & -2 W^{\prime} \smile_{1} \beta-2(\delta \gamma) \smile_{1} \beta-\alpha+U^{\prime} \smile_{2} \beta-\beta \smile_{2} U^{\prime} \\
& +(\delta \beta) \smile_{2} \beta+\beta \smile_{1} \beta+\gamma W^{\prime}+W^{\prime} \gamma+(\delta \gamma) \gamma .
\end{aligned}
$$

A straightforward computation of $4 N+\delta Q$ completes the proof.

We now prove a similar lemma for the cochains of $B$ instead of $A$. The fibre space $\left(A, p_{0}, B, k\right.$-cell) has a cross section $s: B \rightarrow A$. On the cochain level we have

$$
C^{*}(B) \underset{p_{0}^{\sharp}}{\stackrel{s^{\sharp}}{\leftrightarrows}} C^{*}(A)
$$

with $s^{\sharp} \circ p_{0}^{\#}$ the identity.

Lemma 6.2. Let $U \in W_{k} \in H^{k}(B), Z \in P \in H^{2 k-2}(B)$, and let $W$ be any integral cochain representing $W_{k-1}$ for which $\delta W=-2 U$. Then there exist $N \in C^{2 k-2}(B)$ and $Q \in C^{2 k-3}(B)$ such that

$$
4 N+\delta Q=W^{2}+W \smile_{1}(\delta W)-Z-U \smile_{2} U .
$$

Proof. $p_{0}^{\sharp} U, p_{0}^{\sharp} W$, and $p_{0}^{\sharp} Z$ satisfy the conditions of Lemma 6.1. Let $N^{\prime}$ and $Q^{\prime}$ be the cochains of $A$ given by Lemma 6.1 , and $N=s^{\sharp} N^{\prime}$, $Q=s^{\sharp} Q^{\prime}$. Then

$$
\begin{aligned}
& 4 N+\delta Q=s^{\sharp}\left(4 N^{\prime}+\delta Q^{\prime}\right)=\left(s^{\sharp} p_{0}^{\sharp} W\right)^{2} \\
& \quad+s^{\sharp}\left(\left(p_{0}^{\sharp} W\right) \smile_{1}\left(\delta p_{0}^{\sharp} W\right)\right)-s^{\sharp} p_{0}^{\sharp} Z \\
& \quad-s^{\sharp}\left\{\left(p_{0}^{\sharp} U\right) \smile_{2}\left(p_{0}^{\sharp} U\right)\right\}=W^{2}+W \smile_{1}(\delta W)-Z-U \smile_{2} U .
\end{aligned}
$$

We remark that since $4 \delta N=4\left(U \smile_{1} U-W U\right)$ we have $\delta N=$ $U \smile_{1} U-W U$. Also, if $N, Q$ and $N^{\prime}, Q^{\prime}$ satisfy Lemma 6.2 or Lemma 
6.1, then $N-N^{\prime}$ is a cocycle and $4\left(N-N^{\prime}\right)$ is a coboundary, for $4\left(N-N^{\prime}\right)=\delta\left(Q^{\prime}-Q\right)$.

Lemma 6.3. If $\left(U^{\prime}, W^{\prime}, N^{\prime}\right)$ is adjusted, and if $(U, W, N)$ is as in Lemma 6.1, there exist cochains $\beta, \gamma$, and a cocycle $T \in C^{2 k-2}(A)$ such that $4 T$ is a coboundary, $U=U^{\prime}+\delta \beta, W=W^{\prime}+\delta \gamma-2 \beta$,

and

$$
\begin{gathered}
N=N^{\prime}-W^{\prime} \beta+\beta \smile_{1} U^{\prime}+\beta^{2}-U^{\prime} \smile_{1} \beta-(\delta \gamma) \beta \\
+\beta \smile_{1}(\delta \beta)-\gamma U^{\prime}+T .
\end{gathered}
$$

This follows directly from the proof of Lemma 6.1 and the above remark.

Now let $U, W$, and $N$ be any cochains of $B$ which satisfy Lemma 6.2 , and let $M$ be the algebraic mapping cylinder of the map $x \rightarrow x U$, with a product given by Definition 4.2. We then have a product in $H^{*}(M)$. For the remainder of this section, we will use square brackets to denote the natural map $C^{*}(A) \rightarrow C^{*}(A) / C^{*}(A, E)=C^{*}(E)$. The main theorem follows.

TheOREM 6.4. There exist $\eta: M^{p} \rightarrow C^{p}(E)$ an allowable homomorphism and a cocycle $T \in C^{2 k-2}(B)$ such that $4 T$ is a coboundary which have the property that if $(x, y)$ and $(v, w)$ are $p$ and $q$-cocycles, respectively, of $M$, then

$$
\eta\{(x, y)(v, w)\}-\eta(x, y) \eta(v, w)=\left[(-1)^{p+q+1} p_{0}^{\sharp}(y w T)\right]+\delta X
$$

for some cochain $X$ of $E$.

Proof. The homomorphism $\eta$ is defined as follows: Choose $\left(U^{\prime}, W^{\prime}\right.$, $\left.N^{\prime}\right)$ adjusted. We apply Lemma 6.3 to $p_{0}^{\sharp} U, p_{0}^{\sharp} W, p_{0}^{\sharp} N$ to obtain $\beta \in C^{k-1}(A)$ for which $p_{0}^{\sharp} U=U^{\prime}+\delta \beta$. Define $\eta(x, y)=\left[p_{0}^{\sharp} x+p_{0}^{\sharp}(\bar{y}) \cdot \beta\right]$ for $(x, y) \in M$. Then

$$
\begin{aligned}
\delta \eta(x, y) & =\left[\delta p_{0}^{\sharp} x+\left\{\delta p_{0}^{\sharp}(\bar{y})\right\} \beta+p_{0}^{\sharp}(y)(\delta \beta)\right] \\
& =\left[p_{0}^{\sharp} \delta x+p_{0}^{\sharp}(\delta \bar{y}) \beta+p_{0}^{\sharp}(y)\left(p_{0}^{\sharp} U-U^{\prime}\right)\right]
\end{aligned}
$$

while

$$
\begin{aligned}
\eta \delta(x, y) & =\eta(\delta x+y U,-\delta y) \\
& =\left[p_{0}^{\sharp} \delta x+\left(p_{0}^{\sharp} y\right)\left(p_{0}^{\sharp} U\right)+p_{0}^{\sharp}(\overline{-\delta y}) \beta\right] .
\end{aligned}
$$

Since $\overline{-\delta y}=\delta \bar{y}$ and $U^{\prime} \in C^{*}(A, E), \eta \circ \delta=\delta \circ \eta$ and $\eta$ is allowable.

Let $\Gamma=\eta\{(x, y)(v, w)\}-\eta(x, y) \eta(v, w)$, where $(x, y)$ and $(v, w)$ are $p$ and $q$-cocycles respectively of $M$. Then

$$
\begin{aligned}
\Gamma= & {\left[(-1)^{\eta+q} p_{0}^{\sharp}(y)\left(p_{0}^{\sharp} U \smile_{1} p_{0}^{\sharp} v\right)+(-1)^{p+q} p_{0}^{\sharp} y\left\{\left(p_{0}^{\sharp}(w U)\right) \smile_{2} p_{0}^{\sharp} U\right\}\right.} \\
& +(-1)^{p+q+1} p_{0}^{\sharp}(y w)+(-1)^{p} p_{0}^{\sharp}(y v) \beta \\
& +(-1)^{p+q+1}\left(p_{0}^{\sharp} y\right)\left\{\left(p_{0}^{\sharp} w\right) \smile_{1}\left(p_{0}^{\sharp} U\right)\right\} \beta \\
& \left.+(-1)^{p+q+1} p_{0}^{\sharp}(y w W) \beta-\left(p_{0}^{\sharp} \bar{y}\right) \beta\left(p_{0}^{\sharp} v\right)-\left(p_{0}^{\sharp} \bar{y}\right) \beta\left(p_{0}^{\sharp} \bar{w}\right) \beta\right] .
\end{aligned}
$$


We now replace $p_{0}^{\sharp} U$ by $U^{\prime}+\delta \beta$. Note that all terms involving $U^{\prime}$ drop out, for $C^{*}(A, E)$ is an ideal. For simplicity, we write $x^{\prime}=p_{0}^{\sharp}(x)$, etc. Then

$$
\begin{aligned}
\Gamma= & {\left[(-1)^{p+q} y^{\prime}\left((\delta \beta) \smile_{1} v^{\prime}\right)+(-1)^{p+q} y^{\prime}\left\{\left(w^{\prime} \delta \beta\right) \smile_{2}(\delta \beta)\right\}\right.} \\
& +(-1)^{p+q+1} y^{\prime} w^{\prime} p_{0}^{\sharp}(N)+(-1)^{p} y^{\prime} v^{\prime} \beta+(-1)^{p+q+1} y^{\prime}\left(w^{\prime} \smile_{1} \delta \beta\right) \beta \\
& \left.+(-1)^{p+q+1} y^{\prime} w^{\prime} p_{0}^{\sharp}(W) \beta+(-1)^{p+1} y^{\prime} \beta v^{\prime}+(-1)^{p+q+1} y^{\prime} \beta w^{\prime} \beta\right] .
\end{aligned}
$$

Now

$$
\delta\left(\beta \smile_{1} v^{\prime}\right)=(\delta \beta) \smile_{1} v^{\prime}+\beta \smile_{1}\left(\delta v^{\prime}\right)+(-1)^{q+1} \beta v^{\prime}+(-1)^{q} v^{\prime} \beta .
$$

Since $\delta v=-w U, \delta\left(p_{0}^{\sharp} v\right)=-p_{0}^{\sharp} w p_{0}^{\sharp} U=-w^{\prime} U^{\prime}-w^{\prime} \delta \beta$. Thus

$$
\begin{gathered}
(-1)^{p+q} y^{\prime}\left\{(\delta \beta) \smile_{1} v^{\prime}\right\}=(-1)^{p+q} y^{\prime} \delta\left(\beta \smile_{1} v^{\prime}\right)+(-1)^{p+q} y^{\prime}\left(\beta \smile_{1} w^{\prime} U^{\prime}\right) \\
+(-1)^{p+q} y^{\prime}\left\{\beta \smile_{1}\left(w^{\prime} \delta \beta\right)\right\}+(-1)^{p} y^{\prime} \beta v^{\prime}+(-1)^{p+1} y^{\prime} v^{\prime} \beta
\end{gathered}
$$

and

$$
\begin{aligned}
\Gamma= & {\left[(-1)^{p+q} y^{\prime}\left\{\beta \smile_{1}\left(w^{\prime} \delta \beta\right)\right\}+(-1)^{\prime++} y^{\prime}\left\{\left(w^{\prime} \delta \beta\right) \smile_{2}(\delta \beta)\right\}\right.} \\
& +(-1)^{p+q+1} y^{\prime} w^{\prime} p_{0}^{\sharp}(N)+(-1)^{p+q+1} y^{\prime}\left(w^{\prime} \smile_{1} \delta \beta\right) \beta \\
& \left.+(-1)^{p+q+1} y^{\prime} w^{\prime} p_{0}^{\sharp}(W) \beta+(-1)^{p+q+1} y^{\prime} \beta w^{\prime} \beta\right]+ \text { coboundaries },
\end{aligned}
$$

for $y^{\prime}$ is a cocycle and $(-1)^{p+q} y^{\prime} \delta\left(\beta \smile_{1} v^{\prime}\right)$ a coboundary. It is easily checked that

$$
\begin{aligned}
& (-1)^{p+q+1} y^{\prime} \beta w^{\prime} \beta=(-1)^{n} y^{\prime} \delta\left\{\beta \smile_{1} w^{\prime}\right) \beta \\
& \quad+(-1)^{p+1} y^{\prime}\left\{(\delta \beta) \smile_{1} w^{\prime}\right\} \beta+(-1)^{p+q+1} y^{\prime} w^{\prime} \beta^{2} .
\end{aligned}
$$

From Lemma 6.3 we have $p_{0}^{\sharp}(W)=W^{\prime}+\delta \gamma-2 \beta$. From these we get

$$
\begin{aligned}
\Gamma= & {\left[( - 1 ) ^ { p + q } y ^ { \prime } \left\{\beta \smile_{1}\left(w^{\prime} \delta \beta\right)+(-1)^{p+q} y^{\prime}\left\{\left(w^{\prime} \delta \beta\right) \smile_{2}(\delta \beta)\right\}\right.\right.} \\
& +(-1)^{p+q+1} y^{\prime} w^{\prime} p_{0}^{\sharp}(N)+(-1)^{p+q+1} y^{\prime}\left(w^{\prime} \smile_{1} \delta \beta\right) \beta \\
& +(-1)^{p+q+1} y^{\prime} w^{\prime}\left(W^{\prime}+\delta \gamma-2 \beta\right) \beta+(-1)^{p} y^{\prime} \delta\left\{\beta \smile_{1} w^{\prime}\right\} \beta \\
& \left.+(-1)^{p+1} y^{\prime}\left\{(\delta \beta) \smile_{1} w^{\prime}\right\} \beta+(-1)^{p+q+1} y^{\prime} w^{\prime} \beta^{2}\right]+ \text { coboundaries . }
\end{aligned}
$$

Since

$$
\begin{aligned}
& (-1)^{p+1} y^{\prime}\left\{(\delta \beta) \smile_{1} w^{\prime}\right\} \beta+(-1)^{p+q+1} y^{\prime}\left\{w^{\prime} \smile_{1} \delta \beta\right\} \beta \\
& +(-1)^{p} y^{\prime} \delta\left\{\beta \smile_{1} w^{\prime}\right\} \beta=(-1)^{p+1} y^{\prime} \delta\left\{w^{\prime} \smile_{1} \beta\right\} \beta,
\end{aligned}
$$

we have

$$
\begin{aligned}
\Gamma= & {\left[(-1)^{p+q} y^{\prime}\left\{\beta \smile_{1}\left(w^{\prime} \delta \beta\right)\right\}+(-1)^{p+q} y^{\prime}\left\{\left(w^{\prime} \delta \beta\right) \smile_{2} \delta \beta\right\}\right.} \\
& +(-1)^{p+q+1} y^{\prime} w^{\prime} p_{0}^{\sharp}(N)+(-1)^{p+q+1} y^{\prime} w^{\prime}\left(W^{\prime}+\delta \gamma\right) \beta \\
& \left.+(-1)^{p+q} y^{\prime} w^{\prime} \beta^{2}+(-1)^{p+1} y^{\prime} \delta\left\{w^{\prime} \smile_{1} \beta\right\} \beta\right]+ \text { coboundaries . }
\end{aligned}
$$

Now

$$
\begin{aligned}
& (-1)^{p+q} y^{\prime}\left\{\left(w^{\prime} \delta \beta\right) \smile_{2} \delta \beta\right\}=(-1)^{p+1} y^{\prime} \delta\left\{\left(w^{\prime} \delta \beta\right) \smile_{2} \beta\right\} \\
& \quad+(-1)^{p+q+1} y^{\prime}\left\{\left(w^{\prime} \delta \beta\right) \smile_{1} \beta\right\}+(-1)^{p+q+1} y^{\prime}\left\{\beta \smile_{1}\left(w^{\prime} \delta \beta\right),\right.
\end{aligned}
$$


and thus

$$
\begin{aligned}
\Gamma= & (-1)^{p+q+1} y^{\prime}\left\{\left(w^{\prime} \delta \beta \smile_{1} \beta\right\}+(-1)^{p+q+1} y^{\prime} w^{\prime}\left(W^{\prime}+\delta \gamma\right) \beta\right. \\
& +(-1)^{p+q} y^{\prime} w^{\prime} \beta^{2}+(-1)^{p+1} y^{\prime} \delta\left\{w^{\prime} \smile_{1} \beta\right\} \beta \\
& \left.+(-1)^{p+q+1} y^{\prime} w^{\prime}\left\{-W^{\prime} \beta+\beta^{2}-(\delta \gamma) \beta+\beta \smile_{1}(\delta \beta)+T\right\}\right] \\
& + \text { coboundaries, where we have used Lemma } 6.3 \text { on } p_{0}^{\sharp}(N) .
\end{aligned}
$$

Thus

$$
\begin{aligned}
\Gamma= & {\left[(-1)^{p+q+1} y^{\prime}\left\{\left(w^{\prime} \delta \beta\right) \smile_{1} \beta\right\}+(-1)^{p+1} y^{\prime} \delta\left\{w^{\prime} \smile_{1} \beta\right\} \beta\right.} \\
& \left.+(-1)^{p+q+1} y^{\prime} w^{\prime}\left(\beta \smile_{1} \delta \beta\right)+(-1)^{p+q+1} y^{\prime} w^{\prime} T\right]+ \text { coboundaries . }
\end{aligned}
$$

But

$$
(-1)^{p+1} y^{\prime} \delta\left\{w^{\prime} \smile_{1} \beta\right\} \beta=(-1)^{p+1} y^{\prime} \delta\left\{\left(w^{\prime} \smile_{1} \beta\right) \beta\right\}+(-1)^{p+q+1} y^{\prime}\left(w^{\prime} \smile_{1} \beta\right) \delta \beta,
$$

and so

$$
\begin{aligned}
\Gamma= & {\left[(-1)^{p+q+1} y^{\prime}\left\{\left(w^{\prime} \delta \beta\right) \smile_{1} \beta\right\}+(-1)^{p+q+1} y^{\prime}\left(w^{\prime} \smile_{1} \beta\right) \delta \beta\right.} \\
& \left.+(-1)^{p+q+1} y^{\prime} w^{\prime}\left(\beta \smile_{1} \delta \beta\right)+(-1)^{p+q+1} y^{\prime} w^{\prime} T\right]+ \text { coboundaries } \\
= & {\left[( - 1 ) ^ { p + q + 1 } y ^ { \prime } \left\{\left(w^{\prime} \delta \beta\right) \smile_{1} \beta+\left(w^{\prime} \smile_{1} \beta\right) \delta \beta+w^{\prime}\left(\beta \smile_{1} \delta \beta\right)\right.\right.} \\
& \left.+(-1)^{p+q+1} y^{\prime} w^{\prime} T\right]+ \text { coboundaries . }
\end{aligned}
$$

By Hirsch's formula 4.6,

$$
\begin{array}{r}
\Gamma=\left[(-1)^{p+q+1} y^{\prime} w^{\prime}\left\{\beta \smile_{1} \delta \beta+(\delta \beta) \smile_{1} \beta\right\}+(-1)^{p+q+1} y^{\prime} w^{\prime} T\right] \\
+ \text { coboundaries . }
\end{array}
$$

Since $\delta\left(\beta \smile_{1} \beta\right)=\beta \smile_{1} \delta \beta+(\delta \beta) \smile_{1} \beta$,

$$
\Gamma=\left[(-1)^{p+q+1} y^{\prime} w^{\prime} T\right]+\text { coboundaries . }
$$

In view of the fact that $p_{0}^{\#} \circ s^{\#}$ is homotopic to the identity, we have

$$
\Gamma=\left[(-1)^{p+q+1} p_{0}^{\sharp}\left(y w s^{\sharp} T\right)\right]+\text { coboundaries }
$$

as asserted. This completes the proof of Theorem 6.4.

REMARK 6.6. The following diagram commutes except for the square marked " $\otimes$ " which anti-commutes.

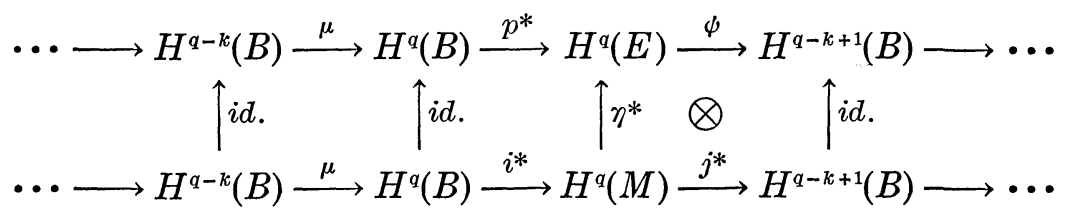

Thus by the five-lemma, $\eta^{*}$ is one-to-one and onto. 
Proof. Let $x^{\prime} \in x \in H^{*}(B)$. Then $\left(x^{\prime}, 0\right)$ represents $i^{*}(x)$ and $\left[p_{0}^{*} x^{\prime}\right]$ represents $\eta^{*} i^{*}(x)$. It also represents $p^{*}(x)$. For the other square, let $(x, y) \in z \in H^{*}(M)$. Then $y$ represents $j^{*}(z)$, and $\left[p_{0}^{\sharp} x+\left(p_{0}^{\sharp} \bar{y}\right) \beta\right]$ represents $\eta^{*}(z)$. Referring to Figure 1, $\psi=p_{0}^{\sharp-1} \theta^{-1} \delta^{*}$. Now $\delta\left\{p_{0}^{\sharp}(x)+\left(p_{0}^{\sharp} \bar{y}\right) \beta\right\}=$ $p_{0}^{\sharp}(-y U)+p_{0}^{\sharp}(y) \delta \beta=-\left(p_{0}^{\sharp} y\right) \cdot U^{\prime}$. Thus $\left(\psi \eta^{*}\right)(z)$ is represented by $-y$.

From equation 6.5 we see that if $H^{2 k-2}(B)$ has no elements of order two, $\eta^{*}: H^{q}(M) \rightarrow H^{q}(E)$ is a ring isomorphism.

Theorem 6.7. Let $\left(E, p, B, S^{k-1}\right)$ and $\left(E^{\prime}, p^{\prime}, B, S^{k-1}\right)$ be two (orientable) $(k-1)$-sphere spaces over the same (compact) base space with $k$ odd. Suppose $H^{2 k-2}(B, Z)$ has no elements of order two. Then if the sphere spaces have the same P-invariant and the same StiefelWhitney classes $W_{k}$ aud $W_{k-1}$, their integral cohomology rings are isomorphic.

To prove this, we observe that both cohomology rings are isomorphic to the cohomology of the mapping cylinder $M$ of Theorem 6.4.

If the rationals or the integers $\bmod n, n$ odd, are used as coefficients for cohomology, then $\eta^{*}$ is always a ring isomorphism since $H^{2 k-2}(B)$ will have no elements of order two. Consequently the cohomology ring with these coefficients of a sphere space is always given by Theorem 6.4 .

7. The case $k$ even. In this section we suppose $\left(E, p, B, S^{k-1}\right)$ is a $(k-1)$-sphere space, with $k$ even. Suppose $V \in C^{k-2}(A)$ is any integral cochain representing $w_{k-2}$. Then for some $W, \delta V=-2 W$ and $W$ represents $w_{k-1}$. Let $U \in w_{k}$. Then $V U$ and $U \smile_{2} U$ both represent $w_{k-2} w_{k}$ and so $V U+U \smile_{2} U$ is a coboundary $\bmod 2$, i.e., there exist $N$ and $Q$ cochains of $A$ for which

$$
2 N+\delta Q=V U+U \smile_{2} U .
$$

From this it follows that $\delta N=U \smile_{1} U-W U$. If also $2 N^{\prime}+\delta Q^{\prime}=$ $V U+U \smile_{2} U$, then $N-N^{\prime}$ is a cocycle and $2\left(N-N^{\prime}\right)$ a coboundary.

Lemma 7.1. Let $(U, W, N)$ be adjusted for the sphere space $\left(E, p, B, S^{k-1}\right)$ and let $V$ be an integral cochain representing $w_{k-2}$ for which $\delta V=-2 W$. Then there exist a cocycle $Y \in C^{k-2}(A)$ and a cochain $X \in C^{2 k-2}(A, E)$ for which

$$
V U+U \smile_{2} U-2 N=2 Y U+\delta X .
$$

Proof. We first remark that it is possible to find such $(U, W, N)$ and $V$. One chooses $V$ to be any integral cochain representing $w_{k-2}$ and defines $W$ by $\delta V=-2 W$. Then $W$ represents $w_{k-1}$. Choose $U \in \mathscr{U} \epsilon$ $H^{k}(A-E)$, and $N \in C^{2 k-2}(A, E)$-such that $\delta N=U \smile_{1} U-W U$. Now let 
$b=V U+U \smile_{2} U-2 N$. Then $b \in C^{2 k-2}(A, E)$ and it is easly seen that $b$ is a cocycle. For some $x, y \in C^{*}(A, E), U \smile_{2} U=V U+2 x+\delta y$ since $U \smile_{2} U$ and $V U$ both represent $w_{k-2} \mathscr{O} \bmod 2$. Thus $b=2(V U+x-N)+\delta y$. Since $b$ is a cocycle, $V U+x-N$ is a cocycle of $C^{*}(A, E)$. The map $\theta$ of (2.1) is an isomorphism, consequently there is a cocycle $Y \in C^{k-2}(A)$ and a cochain $Z \in C^{2 k-2}(A, E)$ such that $V U+x-N=Y U+\delta Z$. Then $b=2 Y U+\delta(2 Z+y)$.

The following crucial lemma may be interpreted as giving a standard form for the cochains $N$ described in the opening paragraphs of this section.

Lemma 7.2. Suppose $U$ is any representative cocycle for $w_{k}, V$ is any cochain representing $w_{k-2}, \delta V=-2 W$, so $W$ represents $w_{k-1}$, $N \in C^{2 k-2}(A), Q \in C^{2 k-3}(A)$, and $2 N+\delta Q=V U+U \smile_{2} U$. Suppose also that $\left(U^{\prime}, W^{\prime}, N^{\prime}\right)$ is adjusted, $V^{\prime}$ represents $w_{k-2}$, and $\delta V^{\prime}=-2 W^{\prime}$. Let $X$ and $Y$ be chosen by Lemma 7.1 so that $V^{\prime} U^{\prime}+U^{\prime} \smile_{2} U^{\prime}-2 N^{\prime}=$ $2 Y U^{\prime}+\delta X$. Then there exist $\beta, \alpha, \gamma$, and $T$, cochains of $A$ of degrees $k-1, k-2, k-3$, and $2 k-2$ respectively so that $T$ is a cocycle, $2 T$ is a coboundary, $U=U^{\prime}+\delta \beta, W=W^{\prime}+\delta \alpha, V=V^{\prime}+\delta \gamma-2 \alpha$, and

$$
N=N^{\prime}-\alpha \delta \beta-\alpha U^{\prime}+(\delta \beta) \smile_{2} U^{\prime}+\beta \smile_{1}(\delta \beta)+\beta^{2}+W^{\prime} \beta+y U^{\prime}+T .
$$

Proof. The existence of $\alpha, \beta$, and $\gamma$ so that the first three equations are satisfied is trivial. To prove the lemma it is only necessary to verify that

$$
\begin{aligned}
& 2\left(N^{\prime}-\alpha \delta \beta-\alpha U^{\prime}+(\delta \beta) \smile_{2} U^{\prime}+\beta \smile_{1}(\delta \beta)+\beta^{2}+W^{\prime} \beta+Y U^{\prime}\right)+\delta Q^{\prime} \\
& \quad=V U+U \smile_{2} U
\end{aligned}
$$

for some cochain $Q^{\prime}$. We choose

$$
Q^{\prime}=\gamma U^{\prime}+V^{\prime} \beta+\gamma \delta \beta+(\delta \beta) \smile_{3} U^{\prime}+\beta \smile_{2}(\delta \beta)+\beta \smile_{1} \beta+X .
$$

The computation is omitted since it is straightforward.

For the next theorem, we return to the cochains of the base space $B$. We suppose $U \in W_{k} \in H^{k}(B), V$ is a cochain representing $W_{k-2} \in$ $H^{k-2}\left(B, Z_{2}\right)$, and $\delta V=-2 W$. Then $W$ represents $W_{k-1} \in H^{k-1}(B)$. We obtain $N$ and $Q$ in $C^{*}(B)$ for which $2 N+\delta Q=V U+U \smile_{2} U$. Let $M$ be the algebraic mapping cylinder of the map $x \rightarrow x U$ for $x \in C^{*}(B)$, with a product given by Definition 4.2. This product satisfies the coboundary formula and induces a product in $H^{*}(M)$. We will use square brackets to denote the natural map $C^{*}(A) \rightarrow C^{*}(A) / C^{*}(A, E)$.

THEOREM 7.3. There exists an allowable homomorphism $\eta: M^{p} \rightarrow$ $C^{p}(E)$ and a cocycle $T \in C^{2 k-2}(B)$ for which $2 T$ is a coboundary with the 
following property: If $(x, y)$ and $(v, w)$ are $p$ and $q$-cocycles, respectively, of $M$, then

$$
\eta\{(x, y)(v, w)\}-\eta(x, y) \eta(v, w)=\left[(-1)^{p} p_{0}^{\sharp}(y w T)\right]+\delta Z
$$

for some cochain $Z$ of $E$.

REMARK 7.5. The following diagram commutes except for the square marked " $\otimes$ " which anti-commutes :

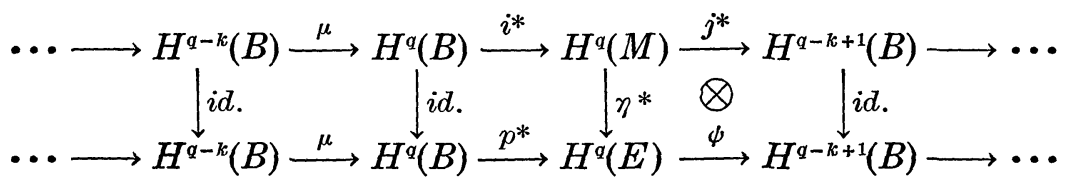

Consequently, by the five-lemma, $\eta^{*}$ is one-to-one and onto.

To prove Theorem 7.3, we first choose $\left(U^{\prime}, W^{\prime}, N^{\prime}\right)$ adjusted and obtain $X$ and $Y$ from Lemma 7.1. Then apply Lemma 7.2 to obtain $\alpha, \beta, \gamma$, and $T$ for which $p_{0}^{\sharp} U=U^{\prime}+\delta \beta, p_{0}^{\sharp} W=W^{\prime}+\delta \alpha, p_{0}^{\sharp} V=V^{\prime}+\delta \gamma-2 \alpha$, and

$$
p_{0}^{\sharp} N=N^{\prime}-\alpha \delta \beta-\alpha U^{\prime}+(\delta \beta) \smile_{2} U^{\prime}+\beta \smile_{1}(\delta \beta)+\beta^{2}+W^{\prime} \beta+Y U^{\prime}+T .
$$

Define, for $(x, y)$ in $M$,

$$
\eta(x, y)=\left[p_{0}^{\sharp} x+\left(p_{0}^{\sharp} \bar{y}\right) \beta\right] .
$$

Then $\eta$ is allowable, i.e., $\delta \eta=\eta \delta$.

The remainder of the proof is omitted, as it is a tedious computation similar to the proof of Theorem 6.4. The proof that the diagram of Remark 7.5 commutes has been given in the proof of Remark 6.6.

From equation (7.4) it follows that if $H^{2 k-2}(B)$ has no elements of order two, $\eta^{*}$ is a ring isomorphism.

Theorem 7.6. Suppose $\left(E, p, B, S^{k-1}\right)$ and $\left(E^{\prime}, p^{\prime}, B, S^{k-1}\right)$ are two (orientable) $(k-1)$-sphere spaces over the same compact base space with $k$ even. Suppose $H^{2 k-2}(B, Z)$ has no elements of order two. Then if the sphere spaces have the same Stiefel-Whitney classes $W_{k}, W_{k-1}$, and $W_{k-2}$, their integral cohomology rings are isomorphic.

This follows because both cohomology rings must be isomorphic to the cohomology ring $H^{*}(M)$.

The following theorem generalizes a result of $\mathrm{R}$. Thom ([4], exposé 17, Théorème 3).

Theorem 7.7. Suppose $\left(E, p, B, S^{k-1}\right)$ is a $(k-1)$-sphere space, for $k$ even. Using the rational numbers or the integers $\bmod n, n$ odd, as 
coefficients for cohomology, the cohomology of the base space and the characteristic class $W_{k}$ determine the cohomology ring of the total space $E$.

Proof. $W_{k-1}=0$ since $2 W_{k-1}=0$. Let $U$ be any representative cocycle for $W_{k} \in H^{k}(B)$, and let $M$ be the algebraic mapping cylinder of $x \rightarrow x U$, for $x \in C^{*}(B)$. We introduce a multiplication in $M$ by choosing $W=0$ and $N=\frac{1}{2}\left(U \smile_{2} U\right)$ or, specifically, the multiplication is defined by

$$
\begin{aligned}
(x, y)(v, w)= & \left(x v+(-1)^{p+q} y\left(U \smile_{1} v\right)+(-1)^{p+1} y\left\{(w U) \smile_{2} U\right\}\right. \\
& \left.+(-1)^{p} y w \frac{1}{2}\left(U \smile_{2} U\right),(-1)^{p} x w+y v+(-1)^{q} y\left(w \smile_{1} U\right)\right)
\end{aligned}
$$

for $(x, y) \in M^{p}$ and $(v, w) \in M^{q}$. Since $\delta \frac{1}{2}\left(U \smile_{2} U\right)=U \smile_{1} U$, this multiplication induces a multiplication in $H^{*}(M)$.

Let $U^{\prime} \in \mathscr{U} \in H^{k}(A-E)$. Then for some $\beta \in C^{k-1}(A), p_{0}^{\sharp} U=U^{\prime}+\delta \beta$. Define $\eta: M^{p} \rightarrow C^{p}(E)$ by $\eta(x, y)=\left[p_{0}^{\sharp} x+\left(p_{0}^{\sharp} \bar{y}\right) \beta\right]$. Then $\eta$ is allowable and induces $\eta^{*}: H^{p}(M) \rightarrow H^{p}(E)$. Let $(x, y)$ and $(v, w)$ be $p$ and $q$ cocycles, respectively, of $M$ and let $\Gamma=\eta\{(x, y)(v, w)\}-\eta(x, y) \eta(v, w)$. Then, letting $x^{\prime}=p_{0}^{\sharp} x$, etc., as before, we have

$$
\begin{aligned}
\Gamma= & {\left[(-1)^{p+q} y^{\prime}\left\{\left(p_{0}^{\sharp} U\right) \smile_{1} v^{\prime}\right\}+(-1)^{p+1} y^{\prime}\left\{\left(w^{\prime} p_{0}^{\sharp} U\right) \smile_{2} p_{0}^{\sharp} U\right\}\right.} \\
& +(-1)^{p} y^{\prime} w^{\prime} \frac{1}{2}\left\{\left(p_{0}^{\sharp} U\right) \smile_{2} p_{0}^{\sharp} U\right\}+(-1)^{p+q+1} y^{\prime} v^{\prime} \beta+(-1)^{p+1} y^{\prime}\left(w^{\prime} \smile_{1} p_{0}^{\sharp} U\right) \beta \\
& \left.+(-1)^{p} y^{\prime} \beta v^{\prime}+(-1)^{p+q+1} y^{\prime} \beta w^{\prime} \beta\right] .
\end{aligned}
$$

Exactly as in the proof of Theorem 6.4, reduce this to

$$
\begin{aligned}
\Gamma= & {\left[(-1)^{p+1} y^{\prime} w^{\prime}\left(\beta \smile_{1} \delta \beta\right)+(-1)^{p} y^{\prime} w^{\prime} \frac{1}{2}\left\{(\delta \beta) \smile_{2} \delta \beta\right\}+(-1)^{p+1} y^{\prime} w^{\prime} \beta^{2}\right] } \\
& + \text { coboundaries } .
\end{aligned}
$$

Since $(\delta \beta) \smile_{2}(\delta \beta)=\delta\left(\beta \smile_{2} \delta \beta\right)+\beta \smile_{1}(\delta \beta)+(\delta \beta) \smile_{1} \beta$,

$$
\begin{aligned}
\Gamma= & {\left[(-1)^{p+1} y^{\prime} w^{\prime} \frac{1}{2}\left(\beta \smile_{1} \delta \beta\right)+(-1)^{p} y^{\prime} w^{\prime} \frac{1}{2}\left(\delta \beta \smile_{1} \beta\right)+(-1)^{p+1} y^{\prime} w^{\prime} \beta^{2}\right] } \\
& + \text { coboundaries }
\end{aligned}
$$

and so $\Gamma$ is a coboundary since

$$
2 \beta^{2}=\delta\left(\beta \smile_{1} \beta\right)+(\delta \beta) \smile_{1} \beta-\beta \smile_{1}(\delta \beta) .
$$

Thus $\eta^{*}$ preserves products. $\eta^{*}$ is shown to be one-to-one and onto exactly as in the proof of Remark 6.6.

In conclusion, we would like to point out that the remarks at the end of Chapter 3 apply also to Theorems 6.7 and 7.6. The question of what one needs to know about $H^{*}(B)$ in addition to the product structure (and various characteristic classes) to determine $H^{*}(E)$ seems to be rather complicated (see [7], Part 1, and [4], exposé 17). Certainly various higher order operations are needed. 


\section{REFERENCES}

1. A. Borel, Topology of Lie groups and characteristic classes, Bull. Amer. Math. Soc. 61 (1955), 397-432.

2. Selected topics in the homology theory of fibre bundles, Department of Mathematics, University of Chicago, 1954, (mimeographed lecture notes).

3. H. Cartan, Espaces fibrés et homotopie, Ecole Normale Supérieure, Paris, 1956 (mimeographed seminar notes).

4. — Algebres d'Eilenberg-MacLane et homotopie, Ecole Normale Supérieure, Paris, 1955 (mimeographed seminar notes).

5. S. Eilenberg and N. E. Steenord, Foundations of algebraic topology, Princeton University Press, 1953.

6. G. Hirsch, Quelques proprietés des produits de Steenrod, C. R. Acad. Sci. Paris 241 (1955), 923-925.

7. W. S. Massey, On the cohomology ring of a sphere bundle, Jour. of Math. and Mech. 7 (1958), 265-290.

8. N. E. Steenrod, The topology of fibre bundles, Princeton University Press, 1951.

9. - Products of cocycles and extensions of mappings, Ann. of Math. 48 (1947), 290-320.

10. Cohomology invariants of mappings, Ann. of Math. 50 (1949), 954-988.

11. R. Thom, Espaces fibrés en spheres et Carrés de Steenrod, Ann. Ecole Norm. Sup. 69 (1952), 109-182.

12. W. T. Wu, On Pontrjagin classes, III, Acta. Math. Sinica 4 (1954), 323-346 (in Chinese, English Summary).

BROWN UNIVERSITY

RUTGers, The State UNIVERSity 


\section{PACIFIC JOURNAL OF MATHEMATICS}

EDITORS

David Gilbarg

Stanford University

Stanford, California

R. A. Beaumont

University of Washington

Seattle 5 , Washington
A. L. Whiteman

University of Southern California

Los Angeles 7, California

L. J. PAIGE

University of California

Los Angeles 24, California

\section{ASSOCIATE EDITORS}

\author{
E. F. BECKENBACH \\ C. E. BURGESS \\ E. HEWITT \\ A. HORN
}

\author{
V. GANAPATHY IYER \\ R. D. JAMES \\ M. S. KNEBELMAN \\ L. NACHBIN
}
I. NIVEN
E. G. STRAUS
T. G. OSTROM
G. SZEKERES
H. L. ROYDEN
F. WOLF
M. M. SCHIFFER
K. YOSIDA

\section{SUPPORTING INSTITUTIONS}

\author{
UNIVERSITY OF BRITISH COLUMBIA \\ CALIFORNIA INSTITUTE OF TECHNOLOGY \\ UNIVERSITY OF CALIFORNIA \\ MONTANA STATE UNIVERSITY \\ UNIVERSITY OF NEVADA \\ OREGON STATE COLLEGE \\ UNIVERSITY OF OREGON \\ OSAKA UNIVERSITY \\ UNIVERSITY OF SOUTHERN CALIFORNIA
}

\author{
STANFORD UNIVERSITY \\ UNIVERSITY OF TOKYO \\ UNIVERSITY OF UTAH \\ WASHINGTON STATE COLLEGE \\ UNIVERSITY OF WASHINGTON \\ $* \quad * \quad *$ \\ AMERICAN MATHEMATICAL SOCIETY \\ CALIFORNIA RESEARCH CORPORATION \\ HUGHES AIRCRAFT COMPANY \\ SPACE TECHNOLOGY LABORATORIES
}

Mathematical papers intended for publication in the Pacific Journal of Mathematics should be typewritten (double spaced), and the author should keep a complete copy. Manuscripts may be sent to any one of the four editors. All other communications to the editors should be addressed to the managing editor, L. J. Paige at the University of California, Los Angeles 24, California.

50 reprints per author of each article are furnished free of charge; additional copies may be obtained at cost in multiples of 50 .

The Pacific Journal of Mathematics is published quarterly, in March, June, September, and December. The price per volume (4 numbers) is $\$ 12.00$; single issues, $\$ 3.50$. Back numbers are available. Special price to individual faculty members of supporting institutions and to individual members of the American Mathematical Society: $\$ 4.00$ per volume; single issues, $\$ 1.25$.

Subscriptions, orders for back numbers, and changes of address should be sent to Pacific Journal of Mathematics, 2120 Oxford Street, Berkeley 4, California.

Printed at Kokusai Bunken Insatsusha (International Academic Printing Co., Ltd.), No. 6, 2-chome, Fujimi-cho, Chiyoda-ku, Tokyo, Japan.

PUBLISHED BY PACIFIC JOURNAL OF MATHEMATICS, A NON-PROFIT CORPORATION

The Supporting Institutions listed above contribute to the cost of publication of this Journal, but they are not owners or publishers and have no responsibility for its content or policies. 


\section{Pacific Journal of Mathematics}

\section{Vol. 9, No. 4 \\ August, 1959}

Frank Herbert Brownell, III, A note on Kato's uniqueness criterion for

Schrödinger operator self-adjoint extensions ............... 953

Edmond Darrell Cashwell and C. J. Everett, The ring of number-theoretic

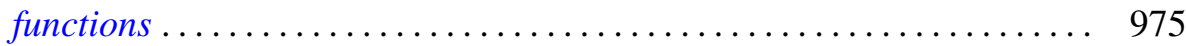

Heinz Otto Cordes, On continuation of boundary values for partial

differential operators ............................. 987

Philip C. Curtis, Jr., n-parameter families and best approximation . . . . . . 1013

Uri Fixman, Problems in spectral operators . . . . . . . . . . . . . . . 1029

I. S. Gál, Uniformizable spaces with a unique structure .............. 1053

John Mitchell Gary, Higher dimensional cyclic elements ............ 1061

Richard P. Gosselin, On Diophantine approximation and trigonometric

polynomials ..................................... 1071

Gilbert Helmberg, Generating sets of elements in compact groups ........ 1083

Daniel R. Hughes and John Griggs Thompson, The H-problem and the

structure of $H$-groups .................................. 1097

James Patrick Jans, Projective injective modules ................. 1103

Samuel Karlin and James L. McGregor, Coincidence properties of birth and

death processes ..................................... 1109

Samuel Karlin and James L. McGregor, Coincidence probabilities ........ 1141

J. L. Kelley, Measures on Boolean algebras ................... 1165

John G. Kemeny, Generalized random variables ................... 1179

Donald G. Malm, Concerning the cohomology ring of a sphere bundle ... . . 1191

Marvin David Marcus and Benjamin Nelson Moyls, Transformations on

tensor product spaces .................................. 1215

Charles Alan McCarthy, The nilpotent part of a spectral operator ........ 1223

Kotaro Oikawa, On a criterion for the weakness of an ideal boundary

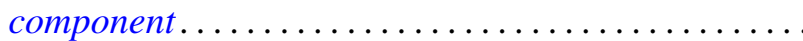

Barrett O'Neill, An algebraic criterion for immersion . . ............... 1239

Murray Harold Protter, Vibration of a nonhomogeneous membrane ... . . . . 1249

Victor Lenard Shapiro, Intrinsic operators in three-space . . . . . . . . . . . 1257

Morgan Ward, Tests for primality based on Sylvester's cyclotomic

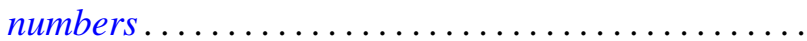

L. E. Ward, A fixed point theorem for chained spaces ....

Alfred B. Willcox, Šilov type $C$ algebras over a connected locally compact

abelian group..................................... 1279

Jacob Feldman, Correction to "Equivalence and perpendicularity of

Gaussian processes" ........................ 